\title{
Synthesis, antimicrobial, and antiproliferative activities of substituted phenylfuranylnicotinamidines
}

This article was published in the following Dove Press journal:

Drug Design, Development and Therapy

II March 2016

Number of times this article has been viewed

\author{
Magdy M Youssef ${ }^{\prime, 2}$ \\ Reem K Arafa ${ }^{3,4}$ \\ Mohamed A Ismail',2 \\ 'Department of Chemistry, College \\ of Science, King Faisal University, \\ Hofuf, Saudi Arabia; ${ }^{2}$ Department \\ of Chemistry, Faculty of Science, \\ Mansoura University, Mansoura, \\ ${ }^{3}$ Department of Pharmaceutical \\ Chemistry, Faculty of Pharmacy, Cairo \\ University, Cairo, ${ }^{4}$ Biomedical Sciences \\ Program, University of Science and \\ Technology, Zewail City of Science \\ and Technology, Cairo, Egypt
}

\begin{abstract}
This research work deals with the design and synthesis of a series of substituted phenylfuranylnicotinamidines $\mathbf{4 a}-\mathbf{i}$. Facile preparation of the target compounds was achieved by Suzuki coupling-based synthesis of the nitrile precursors $\mathbf{3 a}-\mathbf{i}$, followed by their conversion to the corresponding nicotinamidines $\mathbf{4 a}-\mathbf{i}$ utilizing $\mathrm{LiN}(\mathrm{TMS})_{2}$. The antimicrobial activities of the newly synthesized nicotinamidine derivatives were evaluated against the Gram-negative bacterial strains Escherichia coli and Pseudomonas aeruginosa as well as the Gram-positive bacterial strains Staphylococcus aureus and Bacillus megaterium. The minimum inhibitory concentration values of nicotinamidines against all tested microorganisms were in the range of 10-20 $\mu \mathrm{M}$. In specific, compounds $\mathbf{4 a}$ and $\mathbf{4 b}$ showed excellent minimum inhibitory concentration values of $10 \mu \mathrm{M}$ against Staphylococcus aureus bacterial strain and were similar to ampicillin as an antibacterial reference. On the other hand, selected nicotinamidine derivatives were biologically screened for their cytotoxic activities against a panel of 60 cell lines representing nine types of human cancer at a single high dose at National Cancer Institute, Bethesda, MD, USA. Nicotinamidines showing promising activities were further assessed in a five-dose screening assay to determine their compound concentration causing $50 \%$ growth inhibition of tested cell $\left(\mathrm{GI}_{50}\right)$, compound concentration causing $100 \%$ growth inhibition of tested cell (TGI), and compound concentration causing 50\% lethality of tested cell $\left(\mathrm{LC}_{50}\right)$ values. Structure-activity relationship studies demonstrated that the activity of members of this series can be modulated from cytostatic to cytotoxic based on the substitution pattern/nature on the terminal phenyl ring. The most active compound was found to be $4 \mathrm{e}$ displaying a submicromolar $\mathrm{GI}_{50}$ value of $0.83 \mu \mathrm{M}$, with TGI and $\mathrm{LC}_{50}$ values of 2.51 and $100 \mu \mathrm{M}$, respectively. Finally, the possible underlying mechanism of action of this series of compounds was investigated by determining their nuclease-like DNA degradation ability in addition to their antioxidant power and all monocations proved to be effective in all assays.
\end{abstract}

Keywords: substituted phenylfuranylnicotinamidines, Suzuki coupling, antiproliferative, antibacterial, antioxidant

\section{Introduction}

Over the past few years, the development of bacterial resistance to antibiotics has been reported and resistance to multiple antimicrobial agents has become a major health problem. ${ }^{1}$ A continuous discovery of new anti-infectious agents is urgently needed owing to the progress of antibiotic resistance in almost all clinically significant pathogens. ${ }^{2}$ Cancer is a disease that still intrigues the scientific community stimulating their interest in finding novel chemotherapeutic agents effective for the management and remediation of this life-threatening ailment. ${ }^{3}$ Many factors compromise the rate of success of cancer chemotherapy, with the emergence of drug resistance to currently clinically useful agents being the most worrisome in the fight against cancer. ${ }^{4}$ Heterocycle-based chemical entities have been explored as backbone scaffolds for the design of novel antiproliferative
Correspondence: Mohamed A Ismail Department of Chemistry, Faculty of Science, Mansoura University, Mansoura 35516, Egypt

Tel +201096849505

Email mabouelenan@kfu.edu.sa
Drug Design, Development and Therapy 2016:10 ||33-| |46 Dovepress http://dx.doi.org/10.2147/DDDT.S102128 (c) (1) (-) 2016 Youssef et al. This work is published and licensed by Dove Medical Press Limited. The full terms of this license are available at https://www.dovepress.com/terms.php cc) ${ }_{\mathrm{BY}} \mathrm{NC}$ and incorporate the Creative Commons Atribution - Non Commercial (unported, v3.0) License (http:///creativecommons.org/licenses/by-nc/3.0/). By accessing the work you hereby accept the Terms. Non-commercial uses of the work are permitted without any further permission from Dove Medical Press Limited, provided the work is properly attributed. For permission for commercial use of this work, please see paragraphs 4.2 and 5 of our Terms (https://www.dovepress.com/terms.php). 
agents. Pyridine heterocycles are of particular interest because in this class of compounds, the pyridine ring mostly contributes an effective role in the pharmacokinetic and/or the pharmacodynamic properties of these bioactive molecules. Literature has demonstrated the ability of synthetic pyridinecontaining compounds to elicit a wide spectrum of biological activities with emphasis on their usefulness as anticancer therapeutic agents. ${ }^{5}$ Furthermore, furan-containing molecules are found in both natural products and pharmaceuticals; for example, Zantac is one of the most widespread drugs, as well as being on the World Health Organization's list of essential medicines. ${ }^{6}$ On the other hand, the dicationic compound pentamidine has been known for its antimicrobial activity for over 50 years and remains the only aromatic diamidine that is still in clinical use. ${ }^{7,8}$ However, recent studies have shown that pentamidine can also act as an anticancer agent eliciting variable modes of action. ${ }^{9,10}$ Also, furamidine, the furanyl analog of pentamidine, was found to elicit an anticancer activity. ${ }^{11,12}$ Aza-furamidine derivatives have been launched as potent antiprotozoal agents; ${ }^{13}$ from this aza-furamidine series, 2,5-bis(5-amidino-2-pyridyl)furan (DB829; CPD-0802) is a preclinical candidate for treatment of second stage human African trypanosomiasis. ${ }^{14-17}$ Recently, several aryl-2,2'bichalcophene monoamidines, including their aza-analogs, were synthesized and shown to exhibit potent antibacterial, ${ }^{18-20}$ antimutagenic, ${ }^{21,22}$ and anticancer activities. ${ }^{23}$

As part of a research program directed to drug discovery and due to the promising anticancer activities of our recently reported aromatic monoamidines, we have decided to design and synthesize a series of furanylnicotinamidine derivatives, including phenyl, monosubstituted phenyl, and disubstituted phenyl ring analogs. Molecular manipulations have also included both electron donating and electron withdrawing substitutions. The newly synthesized nicotinamidines and one nicotinonitrile compound have been subjected to in vitro cytotoxic evaluation against a panel of 60 cell lines representing nine types of cancer. Finally in this report, antibacterial, DNA cleavage, and antioxidant activities of these novel compounds have also been carried out.

\section{Experimental section Chemistry}

Melting points were recorded using a Gallenhamp melting point apparatus and are uncorrected. Thin-layer chromatography analysis was carried out on silica gel $60 \mathrm{~F}_{254}$ precoated aluminum sheets and detected under ultraviolet light. Infrared (IR) spectra were recorded using KBr wafer technique on a Shimadzu 5800 Fourier transform (FT)-IR spectrometer. ${ }^{1} \mathrm{H}$ NMR spectra were recorded employing a Varian Mercury VX-300 spectrometer and ${ }^{13} \mathrm{C}$ NMR were recorded employing Bruker Avance $400 \mathrm{MHz}$ spectrometer, and chemical shifts $(\delta)$ were in parts per million relative to that of the solvent. Mass spectra were recorded on a gas chromatography mass spectrometry (Schimadzu Qp-2010 Plus) spectrometer. Elemental analyses were performed at the microanalytical laboratories of the Faculty of Science, Cairo University, Cairo, Egypt, on a Perkin-Elmer 2400 analyzer and are within \pm 0.4 of the theoretical values (Table 1 ). 6-(5-Bromofuran-2-yl)nicotinonitrile was prepared as per the reported literature method. ${ }^{13}$

Table I Elemental analysis data

\begin{tabular}{|c|c|c|c|c|c|c|}
\hline \multirow[t]{2}{*}{ Compound } & \multicolumn{3}{|c|}{ Calculated } & \multicolumn{3}{|c|}{ Found } \\
\hline & C & $\mathbf{H}$ & $\mathbf{N}$ & C & $\mathbf{H}$ & $\mathbf{N}$ \\
\hline $3 a\left(\mathrm{C}_{16} \mathrm{H}_{10} \mathrm{~N}_{2} \mathrm{O}\right)$ & 78.03 & 4.09 & 11.38 & 77.81 & 4.17 & 11.19 \\
\hline $\mathbf{3 b}\left(\mathrm{C}_{17} \mathrm{H}_{12} \mathrm{~N}_{2} \mathrm{O}_{2}\right)$ & 73.90 & 4.38 & 10.14 & 73.84 & 4.45 & 10.01 \\
\hline $3 \mathrm{c}\left(\mathrm{C}_{18} \mathrm{H}_{14} \mathrm{~N}_{2} \mathrm{O}_{3}\right)$ & 70.58 & 4.61 & 9.15 & 70.31 & 4.49 & 9.09 \\
\hline $3 \mathbf{d}\left(\mathrm{C}_{18} \mathrm{H}_{15} \mathrm{~N}_{3} \mathrm{O}\right)$ & 74.72 & 5.23 & 14.52 & 74.64 & 5.31 & 14.59 \\
\hline $3 e\left(\mathrm{C}_{16} \mathrm{H}_{9} \mathrm{CIN}{ }_{2} \mathrm{O}\right)$ & 68.46 & 3.23 & 9.98 & 68.61 & 3.14 & 10.12 \\
\hline $3 f\left(\mathrm{C}_{16} \mathrm{H}_{9} \mathrm{FN}_{2} \mathrm{O}\right)$ & 72.72 & 3.43 & 10.60 & 72.57 & 3.50 & 10.47 \\
\hline $3 g\left(\mathrm{C}_{16} \mathrm{H}_{8} \mathrm{Cl}_{2} \mathrm{~N}_{2} \mathrm{O}\right)$ & 60.98 & 2.56 & 8.89 & 61.12 & 2.49 & 8.93 \\
\hline $3 \mathrm{~h}\left(\mathrm{C}_{16} \mathrm{H}_{8} \mathrm{~F}_{2} \mathrm{~N}_{2} \mathrm{O}\right)$ & 68.09 & 2.86 & 9.93 & 68.24 & 2.71 & 9.79 \\
\hline $3 \mathrm{i}\left(\mathrm{C}_{16} \mathrm{H}_{8} \mathrm{~F}_{2} \mathrm{~N}_{2} \mathrm{O}\right)$ & 68.09 & 2.86 & 9.93 & 67.82 & 2.89 & 10.01 \\
\hline $4 \mathrm{a}\left(\mathrm{C}_{16} \mathrm{H}_{13} \mathrm{~N}_{3} \mathrm{O}-2.0 \mathrm{HCl}\right)$ & 57.16 & 4.50 & 12.49 & 57.29 & 4.63 & 12.22 \\
\hline $4 b\left(\mathrm{C}_{17} \mathrm{H}_{15} \mathrm{~N}_{3} \mathrm{O}_{2}-2.0 \mathrm{HCl}-0.75 \mathrm{H}_{2} \mathrm{O}\right)$ & 53.76 & 4.91 & 11.06 & 53.88 & 4.83 & 11.27 \\
\hline $4 \mathrm{c}\left(\mathrm{C}_{18} \mathrm{H}_{17} \mathrm{~N}_{3} \mathrm{O}_{3}-2.0 \mathrm{HCl}-\mathrm{I} .0 \mathrm{H}_{2} \mathrm{O}\right)$ & 52.18 & 5.11 & 10.14 & 52.24 & 4.94 & 10.35 \\
\hline $4 \mathrm{~d}\left(\mathrm{C}_{18} \mathrm{H}_{18} \mathrm{~N}_{4} \mathrm{O}-3.0 \mathrm{HCl}-\mathrm{I} .5 \mathrm{H}_{2} \mathrm{O}\right)$ & 48.82 & 5.46 & 12.65 & 48.50 & 5.23 & 12.37 \\
\hline $4 \mathrm{e}\left(\mathrm{C}_{16} \mathrm{H}_{12} \mathrm{ClN}_{3} \mathrm{O}-2.0 \mathrm{HCl}\right)$ & 51.84 & 3.81 & 11.33 & 52.17 & 3.89 & 11.06 \\
\hline $4 \mathrm{f}\left(\mathrm{C}_{16} \mathrm{H}_{12} \mathrm{FN}_{3} \mathrm{O}-2.0 \mathrm{HCl}-0.5 \mathrm{H}_{2} \mathrm{O}\right)$ & 52.90 & 4.16 & 11.56 & 52.70 & 4.31 & 11.56 \\
\hline $4 \mathrm{~g}\left(\mathrm{C}_{16} \mathrm{H}_{11} \mathrm{Cl}_{2} \mathrm{~N}_{3} \mathrm{O}-2.0 \mathrm{HCl}-0.75 \mathrm{H}_{2} \mathrm{O}\right)$ & 45.90 & 3.49 & 10.04 & 46.10 & 3.46 & 10.20 \\
\hline 4h $\left(\mathrm{C}_{16} \mathrm{H}_{11} \mathrm{~F}_{2} \mathrm{~N}_{3} \mathrm{O}-2.0 \mathrm{HCl}-0.5 \mathrm{H}_{2} \mathrm{O}\right)$ & 50.41 & 3.70 & 11.02 & 50.55 & 3.91 & 11.21 \\
\hline $4 \mathrm{i}\left(\mathrm{C}_{16} \mathrm{H}_{11} \mathrm{~F}_{2} \mathrm{~N}_{3} \mathrm{O}-2.0 \mathrm{HCl}-0.5 \mathrm{H}_{2} \mathrm{O}\right)$ & 50.41 & 3.70 & 11.02 & 50.42 & 3.78 & 11.03 \\
\hline
\end{tabular}


The experiments were conducted under the approval of Deanship of Scientific Research, King Faisal University according to the university regulations (Project No. 150122).

\section{Preparation of (substituted)} phenylfuranylnicotinonitrile derivatives $3 \mathbf{a}-\mathbf{i}$ 6-(5-Phenylfuran-2-yl)nicotinonitrile (3a): To a stirred solution of 6-(5-bromofuran-2-yl)nicotinonitrile $(1.24 \mathrm{~g}$, $5 \mathrm{mmol})$ and $\mathrm{Pd}\left(\mathrm{PPh}_{3}\right)_{4}(150 \mathrm{mg})$ in toluene $(20 \mathrm{~mL})$ was added an aqueous solution of $\mathrm{Na}_{2} \mathrm{CO}_{3}(2 \mathrm{M}, 5 \mathrm{~mL})$, followed by phenylboronic acid $(0.73 \mathrm{~g}, 6 \mathrm{mmol})$ in methanol $(5 \mathrm{~mL})$. The stirred reaction mixture was warmed at $80^{\circ} \mathrm{C}$ for 12 hours, after which the reaction mixture was partitioned between an aqueous solution containing concentrated ammonia $(5 \mathrm{~mL})$ and ethyl acetate $(250 \mathrm{~mL}, 3 \times)$. The organic layer was dried (anhydrous $\mathrm{Na}_{2} \mathrm{SO}_{4}$ ), and then evaporated to dryness under reduced pressure to furnish the nicotinonitrile derivative 3a as a pale-yellow solid in $81 \%$ yield, melting point (mp) $187^{\circ} \mathrm{C}-187.5^{\circ} \mathrm{C}(\mathrm{EtOH} / \mathrm{DMF})$. Rate of flow $\left(\mathrm{R}_{\mathrm{f}}\right)=0.54$, petroleum ether $\left(60^{\circ} \mathrm{C}-80^{\circ} \mathrm{C}\right)$-EtOAc (8:2). IR ( $\left.\mathrm{KBr}\right) v^{\prime} 3,050$, $3,031(\mathrm{CH}), 2,224(\mathrm{CN}), 1,598,1,586,1,551(\mathrm{C}=\mathrm{C}) \mathrm{cm}^{-1} .{ }^{1} \mathrm{H}$ NMR (dimethylsulfoxide [DMSO]- $\left.d_{6}\right) ; \delta 7.25(\mathrm{~d}, \mathrm{~J}=3.3 \mathrm{~Hz}$, 1H), 7.38-7.51 (m, 4H), 7.89-7.92 (m, 2H), 8.07 (d, J=8.4 $\mathrm{Hz}, 1 \mathrm{H}), 8.36(\mathrm{dd}, \mathrm{J}=8.4,2.4 \mathrm{~Hz}, 1 \mathrm{H}), 9.01(\mathrm{~d}, \mathrm{~J}=2.4 \mathrm{~Hz}, 1 \mathrm{H})$. ${ }^{13} \mathrm{C}$ NMR (DMSO- $d_{6}$ ); $\delta 106.8,109.5,115.3,117.8,118.5$, 124.6, 129.1, 129.5, 129.8, 141.2, 151.1, 151.6, 153.3, 156.1 . MS (EI) m/e relative intensity (rel. int.); 246 ( $\left.\mathrm{M}^{+}, 100\right), 218$ (11), 115 (46). Analysis (Anal.) $\left(\mathrm{C}_{16} \mathrm{H}_{10} \mathrm{~N}_{2} \mathrm{O}\right) \mathrm{C}, \mathrm{H}, \mathrm{N}$.

6-[5-(4-Methoxyphenyl)furan-2-yl]nicotinonitrile (3b): Obtained as a golden-yellow solid in $74 \%$ yield, $\mathrm{mp}$ $203^{\circ} \mathrm{C}-204^{\circ} \mathrm{C}, \mathrm{R}_{\mathrm{f}}=0.36$, petroleum ether $\left(60^{\circ} \mathrm{C}-80^{\circ} \mathrm{C}\right)-$ EtOAc (8:2). IR (KBr) v' 3,066, 3,037, 2,983, 2,947 (CH), 2,228 (CN), 1,641, 1,615, 1,598, 1,589 (C=N, C=C) cm ${ }^{-1}$. ${ }^{1} \mathrm{H}$ NMR (DMSO- $\left.d_{6}\right) ; \delta 3.80(\mathrm{~s}, 3 \mathrm{H}), 7.01(\mathrm{~d}, \mathrm{~J}=8.4 \mathrm{~Hz}, 2 \mathrm{H})$, 7.06 (d, J=3.3 Hz, 1H), 7.40 (d, J=3.3 Hz, 1H), 7.81 (d, J=8.4 $\mathrm{Hz}, 2 \mathrm{H}), 8.01$ (d, J=8.4 Hz, 1H), 8.30 (dd, J=8.4, $2.4 \mathrm{~Hz}, 1 \mathrm{H})$, 9.01 (d, J=2.4 Hz, 1H). MS (EI) m/e (rel. int.); $276\left(\mathrm{M}^{+}, 100\right.$ ), 261 (64), 233 (13). Anal. $\left(\mathrm{C}_{17} \mathrm{H}_{12} \mathrm{~N}_{2} \mathrm{O}_{2}\right) \mathrm{C}, \mathrm{H}, \mathrm{N}$.

6-[5-(3,5-Dimethoxyphenyl)furan-2-yl]nicotinonitrile (3c): Obtained as a golden-yellow solid in $80 \%$ yield, $\mathrm{mp}$ $211^{\circ} \mathrm{C}-212.5^{\circ} \mathrm{C} . \mathrm{R}_{\mathrm{f}}=0.30$, petroleum ether $\left(60^{\circ} \mathrm{C}-80^{\circ} \mathrm{C}\right)$-EtOAc (8:2). IR (KBr) v' 3,119, 3,070, 2,993, 2,942 (CH), 2,230 (CN), $1,672,1,591,1,554(\mathrm{C}=\mathrm{N}, \mathrm{C}=\mathrm{C}) \mathrm{cm}^{-1} .{ }^{1} \mathrm{H}$ NMR (DMSO- $\left.d_{6}\right) ; \delta$ $3.80(\mathrm{~s}, 6 \mathrm{H}), 6.50(\mathrm{~s}, 1 \mathrm{H}), 7.01(\mathrm{~s}, 2 \mathrm{H}), 7.23(\mathrm{~d}, \mathrm{~J}=3.3 \mathrm{~Hz}, 1 \mathrm{H})$, 7.39 (d, J=3.3 Hz, 1H), 8.03 (d, J=8.4 Hz, 1H), 8.31 (dd, J=8.4, $2.4 \mathrm{~Hz}, 1 \mathrm{H}), 8.96$ (d, J=2.4 Hz, 1H). MS (EI) m/e (rel. int.); 306 $\left(\mathrm{M}^{+}, 100\right), 277$ (6). Anal. $\left(\mathrm{C}_{18} \mathrm{H}_{14} \mathrm{~N}_{2} \mathrm{O}_{3}\right) \mathrm{C}, \mathrm{H}, \mathrm{N}$.

6-[5-(4-(Dimethylamino)phenyl)furan-2-yl]nicotinonitrile (3d): Obtained as a yellow solid in $77 \%$ yield, $\mathrm{mp} 213^{\circ} \mathrm{C}-214^{\circ} \mathrm{C} . \mathrm{R}_{\mathrm{f}}=0.43$, petroleum ether $\left(60^{\circ} \mathrm{C}-80^{\circ} \mathrm{C}\right)$ EtOAc (8:2). IR (KBr) v' 2,893, 2,808 (CH), 2,221 (CN), $1,615,1,596(\mathrm{C}=\mathrm{N}, \mathrm{C}=\mathrm{C}) \mathrm{cm}^{-1} .{ }^{1} \mathrm{H}$ NMR (DMSO- $\left.d_{6}\right) ; \delta 2.98$ (s, 6H), 6.79 (d, J=8.7 Hz, 2H), 6.93 (d, J=3.6 Hz, 1H), 7.39 (d, J=3.6 Hz, 1H), 7.71 (d, J=8.7 Hz, 2H), 7.93 (d, J=8.4 Hz, 1H), 8.28 (dd, J=8.4, $2.1 \mathrm{~Hz}, 1 \mathrm{H}), 8.94$ (d, J=2.1 Hz, 1H). MS (EI) m/e (rel. int.); 289 (M+1 100$), 274$ (10), 158 (34). Anal. $\left(\mathrm{C}_{18} \mathrm{H}_{15} \mathrm{~N}_{3} \mathrm{O}\right) \mathrm{C}, \mathrm{H}, \mathrm{N}$.

6-[5-(4-Chlorophenyl)furan-2-yl]nicotinonitrile (3e): Obtained as a yellowish-green solid in $80 \%$ yield, $\mathrm{mp}$ $218.5^{\circ} \mathrm{C}-220^{\circ} \mathrm{C} . \mathrm{R}_{\mathrm{f}}=0.50$, petroleum ether $\left(60^{\circ} \mathrm{C}-80^{\circ} \mathrm{C}\right)$ EtOAc (8:2). IR (KBr) v' 3,116 (CH), 2,227 (CN), 1,638, $1,589(\mathrm{C}=\mathrm{N}, \mathrm{C}=\mathrm{C}) \mathrm{cm}^{-1} .{ }^{1} \mathrm{H}$ NMR (DMSO- $\left.d_{6}\right) ; \delta 7.27$ $(\mathrm{d}, \mathrm{J}=3.9 \mathrm{~Hz}, 1 \mathrm{H}), 7.44(\mathrm{~d}, \mathrm{~J}=3.9 \mathrm{~Hz}, 1 \mathrm{H}), 7.54(\mathrm{~d}, \mathrm{~J}=8.4 \mathrm{~Hz}$, 2H), 7.92 (d, J=8.4 Hz, 2H), 8.06 (d, J=8.1 Hz, 1H), 8.35 (dd, J=8.1, $2.1 \mathrm{~Hz}, 1 \mathrm{H}), 8.99$ (d, J=2.1 Hz, 1H). ${ }^{13} \mathrm{C} \mathrm{NMR}$ (DMSO- $\left.d_{6}\right) ; \delta 106.9,110.2,115.3,117.8,118.6,126.3$, 128.7, 129.6, 133.5, 141.3, 151.0, 151.9, 153.3, 154.9. MS (EI) $\mathrm{m} / \mathrm{e}$ (rel. int.); 280, $282\left(\mathrm{M}^{+}, 100,34\right.$ : chlorine isotopes), 252 (17), 149 (45). Anal. $\left(\mathrm{C}_{16} \mathrm{H}_{9} \mathrm{ClN}_{2} \mathrm{O}\right) \mathrm{C}, \mathrm{H}, \mathrm{N}$.

6-[5-(4-Fluorophenyl)furan-2-yl]nicotinonitrile (3f): Obtained as a yellowish-green solid in $76 \%$ yield, $\mathrm{mp} 228^{\circ} \mathrm{C}-$ $229.5^{\circ} \mathrm{C} . \mathrm{R}_{\mathrm{f}}=0.48$, petroleum ether $\left(60^{\circ} \mathrm{C}-80^{\circ} \mathrm{C}\right)-\operatorname{EtOAc}(8: 2)$. IR (KBr) v' 3,139, 3,062 (CH), 2,229 (CN), 1,640, 1,589 $(\mathrm{C}=\mathrm{N}, \mathrm{C}=\mathrm{C}) \mathrm{cm}^{-1} .{ }^{1} \mathrm{H}$ NMR (DMSO- $\left.d_{6}\right) ; \delta 7.21(\mathrm{~d}, \mathrm{~J}=3.6 \mathrm{~Hz}$, 1H), 7.30-7.36 (m, 2H), 7.45 (d, J=3.6 Hz, 1H), 7.93-7.98 (m, 2H), $8.06(\mathrm{~d}, \mathrm{~J}=8.1 \mathrm{~Hz}, 1 \mathrm{H}), 8.36(\mathrm{dd}, \mathrm{J}=8.1,1.8 \mathrm{~Hz}$, 1H), 8.99 (d, J=1.8 Hz, 1H). MS (EI) m/e (rel. int.); 264 (M+, 100), 236 (23), 133 (78). Anal. $\left(\mathrm{C}_{16} \mathrm{H}_{9} \mathrm{FN}_{2} \mathrm{O}\right) \mathrm{C}, \mathrm{H}, \mathrm{N}$.

6-[5-(3,5-Dichlorophenyl)furan-2-yl]nicotinonitrile (3g): Obtained as a brown-yellow solid in 79\% yield, mp $291^{\circ} \mathrm{C}-293^{\circ} \mathrm{C} . \mathrm{R}_{\mathrm{f}}=0.57$, petroleum ether $\left(60^{\circ} \mathrm{C}-80^{\circ} \mathrm{C}\right)-$ EtOAc (8:2). IR (KBr) v' 3,130, 3,060 (CH), 2,230 (CN), $1,642,1,596(\mathrm{C}=\mathrm{N}, \mathrm{C}=\mathrm{C}) \mathrm{cm}^{-1} .{ }^{1} \mathrm{H}$ NMR (DMSO- $\left.d_{6}\right) ; \delta 7.47$ (s, 2H), 7.59 (d, J=3.6 Hz, 1H), 7.97-8.01 (m, 2H), 8.23 (d, J=8.4 Hz, 1H), 8.37 (dd, J=8.4, $2.4 \mathrm{~Hz}, 1 \mathrm{H}), 9.01$ (d, $\mathrm{J}=2.4 \mathrm{~Hz}, 1 \mathrm{H})$. MS (EI) m/e (rel. int.); 314, 315, $316\left(\mathrm{M}^{+}\right.$, 100, 20, 64: two chlorine isotopes), 286 (11), 103 (66). Anal. $\left(\mathrm{C}_{16} \mathrm{H}_{8} \mathrm{Cl}_{2} \mathrm{~N}_{2} \mathrm{O}\right) \mathrm{C}, \mathrm{H}, \mathrm{N}$.

6-[5-(3,5-Difluorophenyl)furan-2-yl]nicotinonitrile (3h): Obtained as a yellowish-green solid in $65 \%$ yield, mp $271^{\circ} \mathrm{C}-273^{\circ} \mathrm{C} . \mathrm{R}_{\mathrm{f}}=0.50$, petroleum ether $\left(60^{\circ} \mathrm{C}-80^{\circ} \mathrm{C}\right)$ EtOAc (8:2). IR (KBr) v' 3,122, 3,091 (CH), 2,230 (CN), $1,661,1,624,1,589(\mathrm{C}=\mathrm{N}, \mathrm{C}=\mathrm{C}) \mathrm{cm}^{-1} .{ }^{1} \mathrm{H}$ NMR $\left(\mathrm{DMSO}-d_{6}\right)$; 反 7.18-7.25 (m, 1H), 7.40 (d, J=3.6 Hz, 1H), 7.45 (d, J=3.6 $\mathrm{Hz}, 1 \mathrm{H}), 7.64-7.69$ (m, 2H), 8.19 (d, J=8.4 Hz, 1H), 8.37 (dd, J=8.4, $2.1 \mathrm{~Hz}, 1 \mathrm{H}), 8.99$ (d, J=2.1 Hz, 1H). MS (EI) $\mathrm{m} / \mathrm{e}$ (rel. int.); $282\left(\mathrm{M}^{+}, 31\right), 80(100)$. Anal. $\left(\mathrm{C}_{16} \mathrm{H}_{8} \mathrm{~F}_{2} \mathrm{~N}_{2} \mathrm{O}\right)$ $\mathrm{C}, \mathrm{H}, \mathrm{N}$. 
6-[5-(2,4-Difluorophenyl)furan-2-yl]nicotinonitrile (3i): Obtained as a pale-yellow solid in 74\% yield, mp $203^{\circ} \mathrm{C}-204.5^{\circ} \mathrm{C} . \mathrm{R}_{\mathrm{f}}=0.52$, petroleum ether $\left(60^{\circ} \mathrm{C}-80^{\circ} \mathrm{C}\right)-$ EtOAc (8:2). IR (KBr) v’ 3,050, 3,031 (CH), 2,224 (CN), $1,598,1,586,1,551(\mathrm{C}=\mathrm{C}) \mathrm{cm}^{-1} \cdot{ }^{1} \mathrm{H}$ NMR (DMSO-d $) ; \delta 7.06$ (d, J=3.9 Hz, 1H), 7.23-7.49 (m, 3H), 8.07-8.15 (m, 2H), 8.38 (dd, J=8.4, $2.1 \mathrm{~Hz}, 1 \mathrm{H}), 9.01$ (d, J=2.1 Hz, 1H). MS (EI) m/e (rel. int.); 282 (M+, 100), 254 (28), 151 (49). Anal. $\left(\mathrm{C}_{16} \mathrm{H}_{8} \mathrm{~F}_{2} \mathrm{~N}_{2} \mathrm{O}\right) \mathrm{C}, \mathrm{H}, \mathrm{N}$.

\section{Preparation of (substituted)}

phenylfuranylnicotinamidine derivatives $\mathbf{4 a - i}$

6-(5-Phenylfuran-2-yl)nicotinamidine hydrochloride salt (4a): The furanylnicotinonitrile derivative 3 a $(638 \mathrm{mg}$, $3 \mathrm{mmol}$ ) was treated with LiN(TMS) ${ }_{2}(1 \mathrm{M}$ solution in tetrahydrofuran [THF], $9 \mathrm{~mL}, 9 \mathrm{mmol}$ ) and the reaction was permitted to stir for 12 hours. The reaction mixture was then cooled, after which hydrogen chloride ethanolic solution (25 $\mathrm{mL}, 1.25 \mathrm{M}$ ) was added, whereupon a precipitate started forming. The mixture was left to run for 8 hours, after which it was diluted with ether and the resultant solid was collected through filtration. The furanylnicotinamidine derivative was purified by neutralization with $1 \mathrm{~N} \mathrm{NaOH}$ followed by filtration of the subsequent solid and washing with water. At the end, the nicotinamidine free base was stirred with hydrogen chloride ethanolic solution for 8 hours, diluted with ether, and the solid formed was filtered and dried to afford the hydrochloride salt of nicotinamidine derivative $4 \mathbf{a}$, as a golden-yellow solid in $72 \%$ yield, mp $261^{\circ} \mathrm{C}-263^{\circ} \mathrm{C}$. IR (KBr) v’ 3,363, 3,200 ( $\left.\mathrm{NH}, \mathrm{NH}_{2}\right), 3,059$ (C-H stretch), 1,677, 1,599, 1,547 (C=N, C=C stretch, $\mathrm{NH}$ bend $) \mathrm{cm}^{-1}$. ${ }^{1} \mathrm{H}$ NMR (DMSO- $\left.d_{6}\right) ; \delta 7.23(\mathrm{~d}, \mathrm{~J}=3.3 \mathrm{~Hz}, 1 \mathrm{H}), 7.38-7.52$ (m, 4H), 7.90-7.93 (m, 2H), 8.10 (d, J=8.4 Hz, 1H), 8.33 (dd, $\mathrm{J}=8.4,2.4 \mathrm{~Hz}, 1 \mathrm{H}), 9.02$ (d, J=2.4 Hz, 1H), 9.32 (br s, 2H, exchangeable with $\mathrm{D}_{2} \mathrm{O}$ ), 9.59 (br s, $2 \mathrm{H}$, exchangeable with $\left.\mathrm{D}_{2} \mathrm{O}\right) .{ }^{13} \mathrm{C}$ NMR (DMSO- $d_{6}$ ); $\delta 109.4,114.8,118.1,122.3$, 124.6, 129.0, 129.5, 129.9, 137.7, 149.8, 151.9, 152.3, 155.9, 164.0. MS (EI) m/e (rel. int.); $263\left(\mathrm{M}^{+}, 100\right), 246\left(\mathrm{M}^{+}-\mathrm{NH}_{3}\right.$, 15), 115 (27). Anal. $\left(\mathrm{C}_{16} \mathrm{H}_{13} \mathrm{~N}_{3} \mathrm{O}-2.0 \mathrm{HCl}\right) \mathrm{C}, \mathrm{H}, \mathrm{N}$.

6-[5-(4-Methoxyphenyl)furan-2-yl]nicotinamidine hydrochloride salt (4b): Obtained as an orange solid in $78 \%$ yield, mp $276^{\circ} \mathrm{C}-278^{\circ} \mathrm{C}$. IR (KBr) v' 3,300, 3,235 ( $\mathrm{NH}, \mathrm{NH}_{2}$ ), 3,083, 3,011, 2,994 (C-H stretch), 1,665, 1,629, 1,602, 1,550 $(\mathrm{C}=\mathrm{N}, \mathrm{C}=\mathrm{C}$ stretch, $\mathrm{NH}$ bend $) \mathrm{cm}^{-1} \cdot{ }^{1} \mathrm{H}$ NMR (DMSO- $\left.d_{6}\right) ; \delta 3.84$ (s, 3H), 7.02 (d, J=8.4 Hz, 2H), 7.07 (d, $\mathrm{J}=3.9 \mathrm{~Hz}, 1 \mathrm{H}), 7.42$ (d, J=3.9 Hz, 1H), 7.83 (d, J=8.4 Hz, 2H), 8.05 (d, J=8.4 Hz, 1H), 8.28 (dd, J=8.4, 2.1 Hz, 1H), 8.97 (d, J=2.1 Hz, 1H), 9.33 (br s, 2H, exchangeable with
$\mathrm{D}_{2} \mathrm{O}$ ), 9.62 (br s, 2H, exchangeable with $\mathrm{D}_{2} \mathrm{O}$ ). ${ }^{13} \mathrm{C}$ NMR $\left(\mathrm{DMSO}-d_{6}\right) ; \delta 55.3,107.3,114.5,114.9,117.5,121.5,122.2$, $125.8,137.4,148.9,150.3,151.4,155.9,159.6,163.4 . \mathrm{MS}$ (EI) m/e (rel. int.); $293\left(\mathrm{M}^{+}, 100\right), 276\left(\mathrm{M}^{+}-\mathrm{NH}_{3}, 17\right)$. Anal. $\left(\mathrm{C}_{17} \mathrm{H}_{15} \mathrm{~N}_{3} \mathrm{O}_{2}-2.0 \mathrm{HCl}-0.75 \mathrm{H}_{2} \mathrm{O}\right) \mathrm{C}, \mathrm{H}, \mathrm{N}$.

6-[5-(3,5-Dimethoxyphenyl)furan-2-yl]nicotinamidine hydrochloride salt (4c): Obtained as an orange solid in 84\% yield, mp $285.5^{\circ} \mathrm{C}-287^{\circ} \mathrm{C}$. IR ( $\left.\mathrm{KBr}\right)$ v' 3,363, 3,200 (NH, $\mathrm{NH}_{2}$ ), 3,059 (C-H stretch), 1,677, 1,599, 1,547 (C=N, C=C stretch, NH bend) $\mathrm{cm}^{-1} .{ }^{1} \mathrm{H}$ NMR (DMSO- $\left.d_{6}\right) ; \delta 3.80(\mathrm{~s}, 6 \mathrm{H})$, $6.51(\mathrm{~s}, 1 \mathrm{H}), 7.03$ (s, 2H), 7.26 (d, J=3.9 Hz, 1H), 7.43 (d, $\mathrm{J}=3.9 \mathrm{~Hz}, 1 \mathrm{H}), 8.10$ (d, J=8.4 Hz, 1H), 8.31 (dd, J=8.4, 2.1 $\mathrm{Hz}, 1 \mathrm{H}), 8.99$ (d, J=2.1 Hz, 1H), 9.31 (br s, 2H, exchangeable with $\mathrm{D}_{2} \mathrm{O}$ ), 9.62 (br s, $2 \mathrm{H}$, exchangeable with $\mathrm{D}_{2} \mathrm{O}$ ). MS (EI) m/e (rel. int.); $323\left(\mathrm{M}^{+}, 100\right), 306\left(\mathrm{M}^{+}-\mathrm{NH}_{3}, 34\right), 286(39) .{ }^{13} \mathrm{C}$ NMR (DMSO- $\left.d_{6}\right) ; \delta 55.5,100.6,102.4,109.7,114.5,117.8$, 122.0, 131.2, 137.4, 149.2, 151.3, 151.6, 155.3, 161.0, 163.6. Anal. $\left(\mathrm{C}_{18} \mathrm{H}_{17} \mathrm{~N}_{3} \mathrm{O}_{3}-2.0 \mathrm{HCl}-1.0 \mathrm{H}_{2} \mathrm{O}\right) \mathrm{C}, \mathrm{H}, \mathrm{N}$.

6-[5-(4-(Dimethylamino)phenyl)furan-2-yl]nicotinamidine hydrochloride salt (4d): Obtained as a brown solid in $66 \%$ yield, $\mathrm{mp} 278^{\circ} \mathrm{C}-280^{\circ} \mathrm{C}$. IR ( $\left.\mathrm{KBr}\right)$ v' 3,390, 3,300 $\left(\mathrm{NH}, \mathrm{NH}_{2}\right.$ ), 3,024, 2,800 (C-H stretch), 1,681, 1,618, 1,596 $(\mathrm{C}=\mathrm{N}, \mathrm{C}=\mathrm{C}$ stretch, $\mathrm{NH}$ bend $) \mathrm{cm}^{-1} .{ }^{1} \mathrm{H} \mathrm{NMR}$ (DMSO- $\left.d_{6}\right)$; $\delta 3.04(\mathrm{~s}, 6 \mathrm{H}), 7.10$ (d, J=3.6 Hz, 1H), 7.20 (br s, 1H, exchangeable with $\left.\mathrm{D}_{2} \mathrm{O}\right), 7.46(\mathrm{~d}, \mathrm{~J}=3.6 \mathrm{~Hz}, 1 \mathrm{H}), 7.86(\mathrm{~d}$, $\mathrm{J}=8.7 \mathrm{~Hz}, 2 \mathrm{H}), 8.07$ (d, J=8.4 Hz, 1H), 8.37 (dd, J=8.4, 2.4 $\mathrm{Hz}, 1 \mathrm{H}), 9.03$ (d, J=2.4 Hz, 1H), 9.42 (br s, 2H, exchangeable with $\mathrm{D}_{2} \mathrm{O}$ ), 9.70 (br s, $2 \mathrm{H}$, exchangeable with $\mathrm{D}_{2} \mathrm{O}$ ). MS (EI) m/e (rel. int.); $306\left(\mathrm{M}^{+}, 100\right), 289\left(\mathrm{M}^{+}-\mathrm{NH}_{3}, 60\right), 158$ (42). Anal. $\left(\mathrm{C}_{18} \mathrm{H}_{18} \mathrm{~N}_{4} \mathrm{O}-3.0 \mathrm{HCl}-1.5 \mathrm{H}_{2} \mathrm{O}\right) \mathrm{C}, \mathrm{H}, \mathrm{N}$.

6-[5-(4-Chlorophenyl)furan-2-yl]nicotinamidine hydrochloride salt (4e): Obtained as a yellow solid in 79\% yield, mp $299^{\circ} \mathrm{C}-301^{\circ} \mathrm{C}$. IR (KBr) v' 3,300, 3,240 (NH, $\left.\mathrm{NH}_{2}\right), 3,016$ (C-H stretch), 1,666, 1,627, 1,600 (C=N, C=C stretch, $\mathrm{NH}$ bend) $\mathrm{cm}^{-1} .{ }^{1} \mathrm{H}$ NMR (DMSO- $\left.d_{6}\right) ; \delta 7.27(\mathrm{~d}$, $\mathrm{J}=3.6 \mathrm{~Hz}, 1 \mathrm{H}), 7.47$ (d, J=3.6 Hz, 1H), 7.55 (d, J=9.0 Hz, 2H), 7.93 (d, J=9.0 Hz, 2H), 8.11 (d, J=8.4 Hz, 1H), 8.37 (dd, J=8.4, $2.4 \mathrm{~Hz}, 1 \mathrm{H}$ ), 9.04 (d, J=2.4 Hz, 1H), 9.42 (br s, $2 \mathrm{H}$, exchangeable with $\mathrm{D}_{2} \mathrm{O}$ ), 9.69 (br s, $2 \mathrm{H}$, exchangeable with $\left.\mathrm{D}_{2} \mathrm{O}\right)$. MS (EI) m/e (rel. int.); 297, $299\left(\mathrm{M}^{+}, 100,36\right.$ : chlorine isotopes), $280\left(\mathrm{M}^{+}-\mathrm{NH}_{3}, 62\right), 149$ (50). ${ }^{13} \mathrm{C} \mathrm{NMR}$ $\left(\mathrm{DMSO}-d_{6}\right) ; \delta 110.4,115.7,119.0,122.7,126.7,128.9$, 130.0, 134.0, 138.4, 149.6, 152.0, 152.3, 155.3, 163.4. Anal. $\left(\mathrm{C}_{16} \mathrm{H}_{12} \mathrm{ClN}_{3} \mathrm{O}-2.0 \mathrm{HCl}\right) \mathrm{C}, \mathrm{H}, \mathrm{N}$.

6-[5-(4-Fluorophenyl)furan-2-yl]nicotinamidine hydrochloride salt (4f): Obtained as a golden-yellow solid in $65 \%$ yield, $\mathrm{mp} 309^{\circ} \mathrm{C}-310.5^{\circ} \mathrm{C}$. IR ( KBr) v' 3,240, 3,170 $\left(\mathrm{NH}, \mathrm{NH}_{2}\right), 3,016(\mathrm{C}-\mathrm{H}$ stretch $), 1,666,1,627,1,604(\mathrm{C}=\mathrm{N}$, 
$\mathrm{C}=\mathrm{C}$ stretch, $\mathrm{NH}$ bend) $\mathrm{cm}^{-1}$. ${ }^{1} \mathrm{H}$ NMR (DMSO- $\left.d_{6}\right) ; \delta 7.21$ $(\mathrm{d}, \mathrm{J}=3.6 \mathrm{~Hz}, 1 \mathrm{H}), 7.31-7.37(\mathrm{~m}, 2 \mathrm{H}), 7.45(\mathrm{~d}, \mathrm{~J}=3.6 \mathrm{~Hz}, 1 \mathrm{H})$, 7.93-7.99 (m, 2H), 8.10 (d, J=8.4 Hz, 1H), 8.36 (dd, J=8.4, $2.4 \mathrm{~Hz}, 1 \mathrm{H}), 9.03(\mathrm{~d}, \mathrm{~J}=2.4 \mathrm{~Hz}, 1 \mathrm{H}), 9.40$ (br s, $2 \mathrm{H}$, exchangeable with $\mathrm{D}_{2} \mathrm{O}$ ), 9.67 (br s, $2 \mathrm{H}$, exchangeable with $\mathrm{D}_{2} \mathrm{O}$ ). MS (EI) $\mathrm{m} / \mathrm{e}$ (rel. int.); $281\left(\mathrm{M}^{+}, 100\right), 264\left(\mathrm{M}^{+}-\mathrm{NH}_{3}, 27\right), 133$ (68). Anal. $\left(\mathrm{C}_{16} \mathrm{H}_{12} \mathrm{FN}_{3} \mathrm{O}-2.0 \mathrm{HCl}-0.5 \mathrm{H}_{2} \mathrm{O}\right) \mathrm{C}, \mathrm{H}, \mathrm{N}$.

6-[5-(3,5-Dichlorophenyl)furan-2-yl]nicotinamidine hydrochloride salt (4g): Obtained as a golden-yellow solid in $80 \%$ yield, $\mathrm{mp} 319^{\circ} \mathrm{C}-321.5^{\circ} \mathrm{C}$. IR ( $\left.\mathrm{KBr}\right) v^{\prime} 3,348$, 3,270 ( $\left.\mathrm{NH}, \mathrm{NH}_{2}\right), 3,085(\mathrm{C}-\mathrm{H}$ stretch), 1,681, 1,597 (C=N, $\mathrm{C}=\mathrm{C}$ stretch, $\mathrm{NH}$ bend) $\mathrm{cm}^{-1}$. ${ }^{1} \mathrm{H}$ NMR (DMSO- $d_{6}$ ); $\delta 7.53$ (s, 2H), 7.54 (s, 1H), 7.97 (s, 2H), 8.23 (d, J=8.4 Hz, 1H), 8.35 (dd, J=8.4, 2.1 Hz, 1H), 9.03 (d, J=2.1 Hz, 1H), 9.40 (br s, $2 \mathrm{H}$, exchangeable with $\mathrm{D}_{2} \mathrm{O}$ ), 9.68 (br s, $2 \mathrm{H}$, exchangeable with $\left.\mathrm{D}_{2} \mathrm{O}\right)$. MS (EI) m/e (rel. int.); 332, 333, $334\left(\mathrm{M}^{+}, 50\right.$, 20, 94: two chlorine isotopes), $315\left(\mathrm{M}^{+}-\mathrm{NH}_{3}, 30\right), 238$ (100). ${ }^{13}$ C NMR (DMSO- $d_{6}$ ); $\delta 111.6,114.2,118.2,122.37,122.41$, 127.4, 132.6, 134.9, 137.3, 149.2, 151.4, 152.3, 152.4, 163.5. Anal. $\left(\mathrm{C}_{16} \mathrm{H}_{11} \mathrm{Cl}_{2} \mathrm{~N}_{3} \mathrm{O}-2.0 \mathrm{HCl}-0.75 \mathrm{H}_{2} \mathrm{O}\right) \mathrm{C}, \mathrm{H}, \mathrm{N}$.

6-[5-(3,5-Difluorophenyl)furan-2-yl]nicotinamidine hydrochloride salt (4h): Obtained as an orange solid in $73 \%$ yield, mp $287.5^{\circ} \mathrm{C}-289^{\circ} \mathrm{C}$. IR ( $\left.\mathrm{KBr}\right)$ v' 3,355, 3,239 (NH, $\mathrm{NH}_{2}$ ), 3,112, 3,077, 3,015 (C-H stretch), 1,667, 1,599, 1,554 $\left(\mathrm{C}=\mathrm{N}, \mathrm{C}=\mathrm{C}\right.$ stretch, $\mathrm{NH}$ bend) $\mathrm{cm}^{-1} .{ }^{1} \mathrm{H}$ NMR (DMSO- $\left.d_{6}\right)$; \& 7.19-7.25 (m, 1H), 7.42 (d, J=3.6 Hz, 1H), 7.47 (d, J=3.6 $\mathrm{Hz}, 1 \mathrm{H}), 7.67-7.70$ (m, 2H), 8.23 (d, J=8.4 Hz, 1H), 8.37 (dd, $\mathrm{J}=8.4,2.4 \mathrm{~Hz}, 1 \mathrm{H}), 9.04(\mathrm{~d}, \mathrm{~J}=2.4 \mathrm{~Hz}, 1 \mathrm{H}), 9.41$ (br s, 2H, exchangeable with $\mathrm{D}_{2} \mathrm{O}$ ), 9.68 (br s, $2 \mathrm{H}$, exchangeable with $\left.\mathrm{D}_{2} \mathrm{O}\right)$. MS (EI) m/e (rel. int.); $299\left(\mathrm{M}^{+}, 52\right), 282\left(\mathrm{M}^{+}-\mathrm{NH}_{3}, 100\right)$, 151 (60). Anal. $\left(\mathrm{C}_{16} \mathrm{H}_{11} \mathrm{~F}_{2} \mathrm{~N}_{3} \mathrm{O}-2.0 \mathrm{HCl}-0.5 \mathrm{H}_{2} \mathrm{O}\right) \mathrm{C}, \mathrm{H}, \mathrm{N}$.

6-[5-(2,4-Difluorophenyl)furan-2-yl]nicotinamidine hydrochloride salt (4i): Obtained as an orange solid in $68 \%$ yield, mp $286^{\circ} \mathrm{C}-288^{\circ} \mathrm{C}$. IR ( $\left.\mathrm{KBr}\right)$ v' 3,359, 3,237 (NH,
$\left.\mathrm{NH}_{2}\right), 3,079,3,014$ (C-H stretch), 1,666, 1,629, 1,603, 1,548 $\left(\mathrm{C}=\mathrm{N}, \mathrm{C}=\mathrm{C}\right.$ stretch, $\mathrm{NH}$ bend) $\mathrm{cm}^{-1} .{ }^{1} \mathrm{H}$ NMR (DMSO- $d_{6}$ ); 反 7.02-7.05 (m, 1H), 7.25-7.44 (m, 2H), 7.47 (d, J=3.6 Hz, $1 \mathrm{H}), 8.07-8.14(\mathrm{~m}, 2 \mathrm{H}), 8.37$ (dd, J=8.4, $2.4 \mathrm{~Hz}, 1 \mathrm{H}), 9.05$ (d, J=2.4 Hz, 1H), 9.43 (br s, 2H, exchangeable with $\mathrm{D}_{2} \mathrm{O}$ ), 9.70 (br s, $2 \mathrm{H}$, exchangeable with $\mathrm{D}_{2} \mathrm{O}$ ). MS (EI) m/e (rel. int.); $299\left(\mathrm{M}^{+}, 68\right), 282\left(\mathrm{M}^{+}-\mathrm{NH}_{3}, 100\right), 151$ (91). Anal. $\left(\mathrm{C}_{16} \mathrm{H}_{11} \mathrm{~F}_{2} \mathrm{~N}_{3} \mathrm{O}-2.0 \mathrm{HCl}-0.5 \mathrm{H}_{2} \mathrm{O}\right) \mathrm{C}, \mathrm{H}, \mathrm{N}$.

\section{Biology}

\section{Antimicrobial activities}

Inhibition zone determination

The antimicrobial activity of the novel nicotinamidine derivatives was verified toward the standard Gram-negative bacterial strains Escherichia coli (E. coli) ATCC 25922 and Pseudomonas aeruginosa (P. aeruginosa) ATCC 27853 and Grampositive bacterial strains Staphylococcus aureus (S. aureus) ATCC 25923 and Bacillus megaterium (B. megaterium) ATCC 14591. The antimicrobial screening was assessed by determining bacterial growth using agar well diffusion technique. ${ }^{24}$ The verified nicotinamidine derivatives $\mathbf{3 d}$ and $\mathbf{4 a -} \mathbf{-}$ were liquefied at $20 \mathrm{mM}$ concentration in DMSO. The LuriaBertani agar (LB) medium was utilized for bacterial growth. ${ }^{25}$ Solutions of the nicotinamidine derivatives equal to $100 \mu \mathrm{M}$ were employed independently in wells, previously cut in the growth medium. The microorganisms were incubated with the tested nicotinamidine derivatives overnight at $37^{\circ} \mathrm{C}$. The subsequent inhibition zones (mm) were recorded (Table 2) and the antimicrobial activities of the tested nicotinamidines against the tested microorganisms were analyzed.

Minimum inhibitory concentrations

The minimum inhibitory concentration (MIC) of nicotinamidine derivatives $\mathbf{3 d}$ and $\mathbf{4 a - i}$ was measured according

Table 2 Effect of the novel furanylnicotinamidine derivatives on Gram-negative and Gram-positive microorganisms

\begin{tabular}{|c|c|c|c|c|}
\hline \multirow[t]{2}{*}{$100 \mu \mathrm{M}-(\mu \mathrm{g} / \mathrm{mL})$} & \multicolumn{2}{|l|}{ Gram-negative } & \multicolumn{2}{|l|}{ Gram-positive } \\
\hline & Escherichia coli & Pseudomonas aeruginosa & Staphylococcus aureus & Bacillus megaterium \\
\hline $4 a-(33.62)$ & 14 & 12 & 12 & 14 \\
\hline 4b- (37.98) & 13 & 11 & 11 & 13 \\
\hline 4c- $(4 I .43)$ & 12 & II & 12 & 14 \\
\hline 3d- (28.93) & 6 & 4 & 4 & 5 \\
\hline 4d- (44.28) & 12 & 11 & 11 & 13 \\
\hline $4 e-(37.07)$ & 13 & 11 & 12 & 13 \\
\hline 4f- $(36.32)$ & 10 & 12 & 10 & 10 \\
\hline 4g- $(41.86)$ & 13 & 12 & 12 & 13 \\
\hline $4 h-(38.12)$ & 11 & II & 12 & 12 \\
\hline $4 i-(38.12)$ & II & 10 & 9 & 10 \\
\hline Ampicillin- (34.94) & 17 & 14 & 13 & 16 \\
\hline
\end{tabular}

Note: The results expressed as zone inhibition in mm diameter. 
Table 3 Minimum inhibitory concentration $\mu \mathrm{M}(\mu \mathrm{g} / \mu \mathrm{L})$ of furanylnicotinamidine derivatives against Gram-negative and Gram-positive microorganisms

\begin{tabular}{llllll}
\hline $\begin{array}{lllll}\text { Compound } \\
\text { number }\end{array}$ & \multicolumn{2}{l}{ Gram-negative $\mu \mathrm{M}(\mu \mathrm{g} / \mu \mathrm{L})$} & & \multicolumn{2}{l}{ Gram-positive $\mu \mathrm{M}(\mu \mathrm{g} / \mu \mathrm{L})$} \\
\cline { 2 - 3 } \cline { 5 - 6 } & Escherichia coli & Pseudomonas aeruginosa & & Staphylococcus aureus & Bacillus megaterium \\
\hline $\mathbf{4 a}$ & $10(3.36)$ & $10(3.36)$ & $15(5.69)$ & $10(3.36)$ & $15(5.40)$ \\
$\mathbf{4 b}$ & $10(3.79)$ & $15(6.21)$ & $15(6.21)$ & $15(5.69)$ \\
$\mathbf{4 c}$ & $15(6.21)$ & $40(11.56)$ & $35(10.12)$ & $15(6.21)$ \\
$\mathbf{3 d}$ & $35(10.12)$ & $15(6.65)$ & $15(6.65)$ & $35(10.12)$ \\
$\mathbf{4 d}$ & $15(6.65)$ & $20(7.41)$ & $20(7.41)$ & $15(6.65)$ \\
$\mathbf{4 e}$ & $15(5.56)$ & $20(7.26)$ & $20(7.26)$ & $15(5.56)$ \\
$\mathbf{4 f}$ & $15(5.45)$ & $15(6.28)$ & $15(6.28)$ & $20(7.26)$ \\
$\mathbf{4 g}$ & $15(6.28)$ & $20(7.62)$ & $20(7.62)$ & $10(4.19)$ \\
$\mathbf{4 h}$ & $15(5.72)$ & $20(7.62)$ & $20(7.62)$ & $15(5.72)$ \\
$\mathbf{4 i}$ & $15(5.72)$ & $5(1.75)$ & $10(3.49)$ & $20(7.62)$ \\
Ampicillin & $5(1.75)$ & &
\end{tabular}

to the processes detailed in Clinical and Laboratory Standards Institute/National Committee for Clinical Laboratory Standards techniques. ${ }^{26}$ Various concentrations $(1-100 \mu \mathrm{M})$ of the tested nicotinamidine derivatives in DMSO were added independently to the previously autoclaved LB liquid medium. The prepared LB cultures E. coli, P. aeruginosa, $S$. aureus, and B. megaterium microorganisms were used for the current assay. All microorganisms were separately cultured in $50 \mu \mathrm{L}$ liquid LB medium having nearly $5 \times 10^{4}$ colony forming units overnight. The tested derivatives were added and the optical density of the examined aliquot was maintained at $\approx 0.5 \mathrm{OD}$ and the test cultures were incubated overnight at $37^{\circ} \mathrm{C}$. Controls (broth alone and culture) were preserved, subsequently. Following the overnight incubation, the optical density of the cultures was measured at $600 \mathrm{~nm}$. The concentration at which the overnight culture (optical density is 0.5 ) turns turbid has been measured as MICs of the examined nicotinamidine samples (Table 3). Experiments were performed as triplicates.

\section{In vitro antiproliferative screening}

Eight of the newly synthesized compounds represented by seven nicotinamidines and one nicotinonitrile were chosen by National Cancer Institute (NCI), Bethesda, MD, USA, for evaluation of their anticancer activity. All selected compounds were subjected to a primary in vitro one-dose $(10 \mu \mathrm{M})$ anticancer assay using the standard NCI 60 cancer cell line panel. The data shown in Table 4 were obtained from the percent growth graphs of treated cells and are presented as mean percent growth inhibition (MPGI). Consequently, compounds displaying promisingly high growth inhibition percentage values were further progressed to the five-dose screen study against the panel of 60 human cancer cell lines, and their compound concentrations causing 50\% growth inhibition of tested cell $\left(\mathrm{GI}_{50}\right)$ values against the individual cell lines are presented in Table 5. Finally, the median $\mathrm{GI}_{50}$, compound concentration causing $100 \%$ growth inhibition of tested cell (TGI) and compound concentration causing 50\% lethality of tested cell $\left(\mathrm{LC}_{50}\right)$ values were determined for the tested monocations against all the 60 tested cell lines as determined from the mean graph midpoints (Table 6).

The experimental procedure used to estimate cell viability or growth was carried out employing a 48-hour continuous drug exposure protocol adopting the sulforhodamine B protein assay and following the previously published standard method. ${ }^{27-29}$

\section{DNA binding and degradation assay}

The DNA binding and degradation test of the nicotinamidine derivatives was done as described. ${ }^{30}$ Briefly, the tested nicotinamidines were liquefied in DMSO $(20 \mathrm{mM})$ and $0.5,1,2$, 3 , and $4 \mu \mathrm{M}$ were added individually to $2 \mu \mathrm{g}$ DNA purified from E. coli. The controls for this test were used as DNA alone and DNA in DMSO. The assays were carried out at $37^{\circ} \mathrm{C}$ for 1 hour and the DNA degradation was evaluated via agarose gel electrophoresis..$^{31}$ Ethidium bromide was

Table 4 In vitro MPGI of the novel furanylnicotinamidine derivatives against a panel of 60 cell lines at a single dose level (I0 $\mu M)(N C l$, Bethesda, MD, USA)

\begin{tabular}{|c|c|c|c|c|c|c|c|c|}
\hline Compound number & 3d & $4 a$ & $4 b$ & 4c & 4d & $4 e$ & $4 f$ & $4 g$ \\
\hline MPGI & 25.48 & 20.02 & 33.75 & -30.47 & -56.15 & -74.08 & -52.59 & -49.88 \\
\hline
\end{tabular}

Abbreviations: MPGI, mean percent growth inhibition; NCl, National Cancer Institute. 
Table 5 In vitro antiproliferative activity of the novel furanylnicotinamidines against a panel of 60 cell lines at a five-dose level (National Cancer Institute, Bethesda, MD, USA)

\begin{tabular}{|c|c|c|c|c|c|c|}
\hline Cancer type/cell line & $4 b$ & $4 c$ & 4d & $4 e$ & $4 f$ & $4 g$ \\
\hline \multicolumn{7}{|l|}{ Leukemia } \\
\hline CCRF-CEM & 3.20 & 2.06 & 1.80 & 0.856 & 1.51 & 1.93 \\
\hline HL-60 (TB) & 2.14 & 2.12 & 1.76 & 0.219 & 1.52 & 2.42 \\
\hline K-562 & 3.97 & 1.32 & 0.541 & 0.314 & 0.934 & 1.50 \\
\hline MOLT-4 & 4.40 & 1.74 & 1.59 & 0.681 & 1.89 & 2.46 \\
\hline RPMI-8226 & 2.78 & 1.92 & 1.30 & 0.310 & 1.20 & 2.03 \\
\hline SR & 3.11 & 1.73 & 0.378 & 0.275 & 1.32 & $\mathrm{I} .78$ \\
\hline \multicolumn{7}{|c|}{ Non-small cell lung cancer } \\
\hline A549/ATCC & 5.10 & 1.86 & 1.89 & 1.06 & 1.50 & 1.90 \\
\hline HOP-62 & 10.5 & 1.70 & 1.74 & 1.35 & 1.50 & 1.98 \\
\hline HOP-92 & 1.31 & 1.42 & 1.27 & $0.40 \mathrm{I}$ & 0.579 & 1.56 \\
\hline $\mathrm{NCl}-\mathrm{H} 226$ & 12.9 & 1.81 & 1.88 & 1.52 & 1.40 & 2.13 \\
\hline $\mathrm{NCl}-\mathrm{H} 23$ & 11.6 & 1.90 & 1.77 & 1.40 & 1.76 & 1.96 \\
\hline $\mathrm{NCl}-\mathrm{H} 322 \mathrm{M}$ & 6.00 & 1.60 & $\mathrm{I} .44$ & 0.838 & 1.73 & 1.74 \\
\hline $\mathrm{NCl}-\mathrm{H} 460$ & 3.69 & 1.63 & 1.35 & 0.293 & 1.61 & 1.90 \\
\hline $\mathrm{NCl}-\mathrm{H} 522$ & 6.80 & 1.73 & 1.84 & 1.61 & 1.51 & 1.98 \\
\hline \multicolumn{7}{|l|}{ Colon cancer } \\
\hline Colo 205 & 1.82 & 1.62 & 1.04 & 0.201 & 0.817 & 2.01 \\
\hline HCC-2998 & 2.45 & 1.69 & 1.07 & 0.212 & 1.22 & 1.92 \\
\hline HCT-II6 & 3.26 & 1.66 & $\mathrm{I} .47$ & 0.405 & 1.40 & 1.97 \\
\hline HCT-I5 & 4.62 & 1.84 & 1.36 & 0.486 & 1.30 & 1.80 \\
\hline НT29 & 3.40 & 1.39 & 0.479 & 0.319 & 0.736 & 1.76 \\
\hline $\mathrm{KMI} 2$ & 4.41 & 1.78 & 1.43 & 0.388 & 1.69 & 1.85 \\
\hline SW-620 & 4.05 & 1.67 & 0.774 & 0.492 & 1.61 & 1.78 \\
\hline \multicolumn{7}{|l|}{ CNS cancer } \\
\hline SF-268 & 11.2 & 1.86 & 1.77 & 1.30 & 1.55 & 1.82 \\
\hline SF-295 & NT & 1.86 & 2.00 & 1.79 & 1.54 & 1.92 \\
\hline SF-539 & 4.74 & 1.55 & 1.54 & 1.19 & $\mathrm{I} .4 \mathrm{I}$ & 1.76 \\
\hline SNB-19 & 11.7 & 1.85 & 1.87 & 1.56 & 1.72 & 1.63 \\
\hline SNB-75 & 10.5 & 1.48 & 1.59 & 1.35 & 1.24 & 1.64 \\
\hline U25I & 4.70 & 1.62 & 1.53 & 1.28 & 1.49 & 1.76 \\
\hline \multicolumn{7}{|l|}{ Melanoma } \\
\hline LOX IMVI & 2.58 & 1.63 & 1.53 & 0.306 & $\mathrm{I} .48$ & 1.77 \\
\hline MALME-3M & 3.25 & 1.82 & 2.04 & 1.35 & 1.94 & 2.42 \\
\hline $\mathrm{MI} 4$ & 2.12 & 1.90 & 1.85 & 1.31 & 1.54 & 1.90 \\
\hline MDA-MB-435 & 2.18 & 1.55 & 1.24 & 0.381 & 1.36 & 1.76 \\
\hline SK-MEL-2 & 4.91 & 2.01 & 1.96 & 1.95 & 2.02 & 2.09 \\
\hline SK-MEL-28 & 8.23 & 1.65 & 1.76 & 1.52 & 1.59 & 1.80 \\
\hline SK-MEL-5 & 3.43 & 1.60 & 1.58 & 1.48 & 1.46 & 1.77 \\
\hline UACC-257 & 2.18 & 1.76 & 1.77 & 1.31 & 1.71 & 1.84 \\
\hline UACC-62 & 10.3 & 1.77 & 1.76 & 1.57 & 1.69 & 1.78 \\
\hline \multicolumn{7}{|l|}{ Ovarian cancer } \\
\hline IGROV-I & 5.29 & 1.81 & 1.89 & 1.50 & 1.70 & 2.09 \\
\hline OVCAR-3 & 8.90 & 1.89 & 1.75 & 1.34 & 1.87 & 1.88 \\
\hline OVCAR-4 & 10.4 & 1.74 & 1.56 & 1.17 & 1.40 & 1.90 \\
\hline OVCAR-5 & 10.9 & 1.74 & 1.62 & 1.19 & 1.58 & 1.73 \\
\hline OVCAR-8 & 11.3 & I.7I & 1.74 & 1.67 & 1.70 & 1.88 \\
\hline $\mathrm{NCl} / \mathrm{ADR}-\mathrm{RES}$ & 14.1 & 6.53 & 2.03 & 1.63 & 1.69 & 1.93 \\
\hline SK-OV-3 & 10.2 & 1.91 & 1.88 & 1.53 & 1.40 & 1.95 \\
\hline \multicolumn{7}{|l|}{ Renal cancer } \\
\hline $786-0$ & 6.02 & 1.72 & 1.79 & 1.27 & 1.46 & 1.95 \\
\hline A498 & 12.8 & 1.22 & 1.09 & 0.770 & 1.26 & 1.47 \\
\hline $\mathrm{ACHN}$ & 6.56 & 1.71 & 1.75 & 1.23 & 1.66 & 1.81 \\
\hline CAKI-I & 6.79 & NT & NT & NT & 1.30 & 1.76 \\
\hline RXF 393 & 7.01 & 1.60 & 1.58 & 1.16 & 1.19 & 1.73 \\
\hline
\end{tabular}

(Continued)
Table 5 (Continued)

\begin{tabular}{|c|c|c|c|c|c|c|}
\hline Cancer type/cell line & $4 b$ & 4c & 4d & $4 e$ & $4 f$ & $4 g$ \\
\hline $\mathrm{SNI} 2 \mathrm{C}$ & 8.01 & 1.62 & 1.58 & 1.23 & 1.69 & 1.62 \\
\hline TK-10 & 10.7 & 1.79 & 1.95 & 1.94 & 1.74 & 1.87 \\
\hline UO-3I & 2.68 & 1.59 & 1.48 & 0.960 & 1.60 & 1.54 \\
\hline \multicolumn{7}{|l|}{ Prostate cancer } \\
\hline PC-3 & 4.18 & 1.65 & 1.50 & 1.03 & 1.48 & 1.74 \\
\hline DU-I45 & 11.3 & 1.76 & 1.72 & 0.834 & 1.70 & 1.87 \\
\hline \multicolumn{7}{|l|}{ Breast cancer } \\
\hline MCF-7 & 3.17 & 1.74 & 1.61 & 0.484 & 1.37 & 1.73 \\
\hline MDA-MB-23I/ATCC & 3.34 & 1.83 & 1.80 & 0.469 & NT & 1.65 \\
\hline HS-578T & 10.9 & 1.66 & 1.90 & 1.31 & 1.57 & 1.87 \\
\hline BT-549 & 10.7 & 1.78 & 1.80 & 1.56 & 1.40 & 1.89 \\
\hline $\mathrm{T}-47 \mathrm{D}$ & 5.13 & 1.72 & 1.73 & 0.379 & NT & 2.18 \\
\hline MDA-MB-468 & 2.04 & 1.51 & 1.39 & 0.507 & 1.37 & 1.69 \\
\hline
\end{tabular}

Note: Data represent the compounds' $\mathrm{Gl}_{50}$ in $\mu \mathrm{M}$ against the tested cell lines.

Abbreviations: $\mathrm{Gl}_{50}$, drug concentration that causes $50 \%$ inhibition of cell growth; NT, not tested; CNS, central nervous system.

used to stain agarose gels, which visualized by an ultraviolet transilluminator.

\section{Antioxidant activity}

Super oxide dismutase (SOD) mimetic catalytic activity assay

The SOD-like activity of the tested furanylnicotinamidines was measured via nitroblue tetrazolium (NBT)/reduced nicotinamide adenine dinucleotide (NADH)/phenazine methosulfate to photo-generate $\mathrm{O}_{2}{ }^{--}$in phosphate buffer $(\mathrm{pH}=8.3)$. The reduced NBT to blue formazan was utilized as a marker of $\mathrm{O}_{2}^{--}$generation and detected at $560 \mathrm{~nm}$. The reaction in the presence of the nicotinamidine derivatives and controls was recorded for 5 minutes. ${ }^{32}$ The percentages of inhibition of free radical generation from SOD-like activity were measured via the equation:

$$
\mathrm{I} \%=\frac{\Delta \mathrm{A}_{\text {control }}-\Delta \mathrm{A}_{\text {sample }}}{\Delta \mathrm{A}_{\text {control }}} \times 100
$$

where $\mathrm{A}_{\text {control }}$ is the absorbance of the control (comprising all reagents except the nicotinamidine derivatives) and $\mathrm{A}_{\text {sample }}$ is the absorbance of the nicotinamidine derivatives.

Table 6 Median $\mathrm{Gl}_{50}, \mathrm{TGl}$, and $\mathrm{LC}_{50}(\mu \mathrm{M})$ for the most active furanylnicotinamidines against a panel of 60 cell lines at a five-dose level ( $\mathrm{NCl}$, Bethesda, MD, USA)

\begin{tabular}{lllllll}
\hline MG-MID & 4b & 4c & 4d & 4e & 4f & $\mathbf{4 g}$ \\
\hline $\mathrm{GI}_{50}$ & 5.24 & $\mathrm{I} .66$ & $\mathrm{I} .5 \mathrm{I}$ & 0.83 & $\mathrm{I} .44$ & $\mathrm{I} .86$ \\
$\mathrm{TGI}$ & $\mathrm{I} 5.48$ & 3.47 & 3.16 & $2.5 \mathrm{I}$ & 2.95 & 3.63 \\
$\mathrm{LC}_{50}$ & 43.65 & 7.58 & 7.24 & 100 & 6.02 & 7.94 \\
\hline
\end{tabular}

Abbreviations: MG-MID, mean graph midpoint representing mean sensitivity of all examined cell lines to the test compound; $\mathrm{Gl}_{50}$, compound concentration causing $50 \%$ growth inhibition of tested cells; TGl, compound concentration causing $100 \%$ growth inhibition of tested cells; $\mathrm{LC}_{50}$, compound concentration causing $50 \%$ lethality of tested cells; $\mathrm{NCl}$, National Cancer Institute. 


\section{DPPH free radical scavenging activity}

The 2,2-diphenyl-1- picrylhydrazyl (DPPH) free radical scavenging activities of furanylnicotinamidine derivatives were carried out as reported by Al-Omair et al. ${ }^{33}$ The reduction of stable DPPH free radical from a purple to a yellow colored diphenylpicryl hydrazine was measured by the electron donor competence of the tested nicotinamidine derivatives. After an electron was moved to the odd electron in DPPH•, the absorbance at $517 \mathrm{~nm}$ reduced steadily to increase nonradical DPPH forms. Briefly, $1 \mathrm{~mL}$ of the test compound $(2 \mu \mathrm{M})$ in ethanol/DMSO $(1: 1 \mathrm{v}: \mathrm{v})$ or standard (vitamin C) was added to $4 \mathrm{~mL}$ of $0.004 \%$ (w/v) ethanol solution of DPPH and vortexed carefully. After a 30-minute incubation at $30^{\circ} \mathrm{C}$, the absorbance was recorded against control (methanol/DMSO 1:1 v:v) at $517 \mathrm{~nm}$. Consequently, after an electron was transferred to the odd electron in DPPH•, the absorbance at $517 \mathrm{~nm}$ reduced steadily due to the increase of the nonradical DPPH forms. The percentage of inhibition of DPPH free radical was calculated by the equation:

$$
\mathrm{I} \%=\frac{\mathrm{A}_{\text {control }}-\mathrm{A}_{\text {sample }}}{\mathrm{A}_{\text {control }}} \times 100
$$

where $\mathrm{A}_{\text {control }}$ is the absorbance of the control (comprising all substances except the nicotinamidine derivatives) and $\mathrm{A}_{\text {sample }}$ is the absorbance of the furanylnicotinamidine derivatives.

\section{NO scavenging assay}

The scavenging activity method of nitric oxide (NO) was achieved according to the assay of Green et $\mathrm{al}^{34}$ where sodium nitroprusside generates NO radicals, which cooperate with oxygen to generate nitrite ions. The latter can be evaluated by utilizing the Griess reagent $\left(2 \% \mathrm{H}_{3} \mathrm{PO}_{4}, 1 \%\right.$ sulfanilamide, and $0.1 \% \mathrm{~N}-(1-$ naphthyl)ethylenediamine dihydrochloride). The NO scavengers participate with $\mathrm{O}_{2}$ to minimize the generation of nitrite ions. Sodium nitroprusside $(10 \mathrm{mM})$ in phosphate buffer (PB) saline was mixed with the tested nicotinamidine derivatives $(2 \mu \mathrm{M})$ and standard. The reaction mixtures were kept for 2.5 hours at $25^{\circ} \mathrm{C}$ after which Griess reagent $(0.5 \mathrm{~mL})$ was added..$^{35}$ The absorbance of the pink color generated was read at $546 \mathrm{~nm}$ and referred to the absorbance of standard solutions of $\mathrm{NaNO}_{2}$ treated in the same way with Griess reagent. NO scavenging activity was considered according to the equation:

$$
\% \text { NO scavenging activity }=\frac{A_{\text {control }}-A_{\text {sample }}}{A_{\text {control }}} \times 100
$$

where $\mathrm{A}_{\text {control }}$ is the absorbance of the control (comprising all reagents except the nicotinamidine derivatives) and $\mathrm{A}_{\text {sample }}$ is the absorbance of the nicotinamidine derivatives.

All data about total antioxidant activity are the average of triplicate analyses. All procedures were approved by the University of King Faisal Committee of Scientific Research Ethics.

\section{Results and discussion}

\section{Chemistry}

The syntheses of the novel furanylnicotinamidines $\mathbf{4 a - i}$ were initiated by the Suzuki coupling reaction of 6-(5-bromofuran2-yl)nicotinonitrile with the corresponding phenylboronic<smiles>N#Cc1ccc(-c2ccc(Br)o2)nc1</smiles>

1

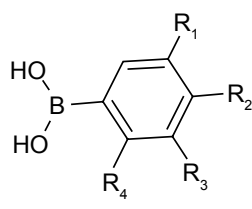

2a-i

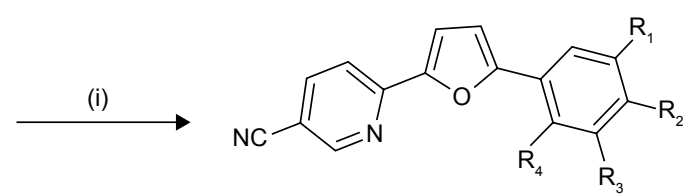

3a-i

$\begin{array}{rllll}\text { Code: } & \mathbf{R}_{1} & \mathbf{R}_{2} & \mathbf{R}_{3} & \mathbf{R}_{4} \\ \text { 2, 3, 4a } & \mathrm{H} & \mathrm{H} & \mathrm{H} & \mathrm{H} \\ \mathbf{b} & \mathrm{H} & \mathrm{OCH}_{3} & \mathrm{H} & \mathrm{H} \\ \mathbf{c} & \mathrm{OCH}_{3} & \mathrm{H} & \mathrm{OCH}_{3} & \mathrm{H} \\ \text { d } & \mathrm{H} & \mathrm{N}(\mathrm{Me})_{2} & \mathrm{H} & \mathrm{H} \\ \mathbf{e} & \mathrm{H} & \mathrm{Cl} & \mathrm{H} & \mathrm{H} \\ \mathbf{f} & \mathrm{H} & \mathrm{F} & \mathrm{H} & \mathrm{H} \\ \mathbf{g} & \mathrm{Cl} & \mathrm{H} & \mathrm{Cl} & \mathrm{H} \\ \mathbf{h} & \mathrm{F} & \mathrm{H} & \mathrm{F} & \mathrm{H} \\ \mathbf{i} & \mathrm{H} & \mathrm{F} & \mathrm{H} & \mathrm{F}\end{array}$

$4 a-i$

Figure I Synthesis scheme for the new furanylnicotinamidine derivatives.

Notes: (i) $\mathrm{Pd}\left(\mathrm{PPh}_{3}\right)_{4}, \mathrm{Na}_{2} \mathrm{CO}_{3}$, toluene, $80^{\circ} \mathrm{C}$; (ii) a) $\mathrm{LiN}(\mathrm{TMS})_{2}$, THF, r.t., overnight, b) $\mathrm{HCl}$ (gas), dry ethanol, r.t., overnight.

Abbreviation: r.t., room temperature. 
acid derivatives to furnish the phenylfuranylnicotinonitrile derivatives 3a-i (Figure 1). The nicotinonitriles were converted to the corresponding nicotinamidine derivatives by the action of LiN(TMS) $)_{2}$ solution in THF. The structures of the newly synthesized nicotinamidine derivatives $\mathbf{4} \mathbf{a}-\mathbf{i}$ and $\mathbf{3 a}-\mathbf{i}$ were established based on their spectral and elemental analyses. Thus, ${ }^{1} \mathrm{H}$ NMR spectrum of the nicotinamidine derivative $\mathbf{4 b}$ displayed two deuterium exchangeable singlet signals at $\delta 9.33(2 \mathrm{H})$ and $\delta 9.62(2 \mathrm{H})$ characteristic for the cationic amidine group in addition to the signals corresponding to the 1,4-disubstituted benzene ring, furan, and 2,6-disubstituted pyridyl moieties. ${ }^{13} \mathrm{C}$ NMR spectrum showed 15 carbon lines that assigned to the carbon network of compound $\mathbf{4 b}$ structure. Furthermore, mass spectrum of nicotinamidine $\mathbf{4 b}$ furnished an $\mathrm{m} / \mathrm{z}$ peak at 293 of its molecular ion peak $\left(\mathrm{M}^{+}\right)$.

\section{Biology}

Antimicrobial activities

Inhibition zone determination

The nicotinamidines $\mathbf{4} \mathbf{a}-\mathbf{i}$ and nicotinonitrile derivative 3d were tested against the growth of both Gram-negative and Gram-positive microorganisms. The zone of inhibition diameter for the tested nicotinamidine derivatives against the growth of the verified microorganisms is presented in Table 2. From these results, it is apparent that the nicotinamidine derivatives $4 \mathbf{a}, \mathbf{4 b}, \mathbf{4 c}, \mathbf{4 d}, \mathbf{4 e}$, and $\mathbf{4 g}$ exhibit the most antimicrobial activity toward the wholly considered microorganisms compared to ampicillin as an antibacterial control. The antimicrobial activity of the nicotinamidine derivatives that have electron donating groups in the phenyl ring as $p-\mathrm{OCH}_{3}, 3,5$-dimethoxy, and $p-\mathrm{N}\left(\mathrm{CH}_{3}\right)_{2}$ in compounds $\mathbf{4 b} \mathbf{b} \mathbf{d}$ is lower than that recorded with the parent compound 4a. This finding was also the case for the nicotinamidine derivatives that have electron withdrawing atoms substituents on the phenyl ring. The nicotinamidine compounds $\mathbf{4 f}, \mathbf{4 h}$, and $4 \mathbf{i}$ reveal good antimicrobial activity toward the examined microorganisms, but still less than the parent compound 4a. The nicotinamidines $\mathbf{4 e}$ and $\mathbf{4 g}$ substituted with chloro/ dichlorophenyl ring have greater antibacterial activity than their fluoro/difluoro counterparts $\mathbf{4 f}, \mathbf{4 h}$, and $\mathbf{4 i}$. However, the nicotinonitrile derivative $\mathbf{3 d}$ reveals the lowest antimicrobial activity of all, over the study. From a structure point of view, it is noticeable that the existence of amidine group in compounds 4a-i increased the antimicrobial activities of the nicotinamidines compared to the nicotinonitrile derivative $\mathbf{3 d}$. Due to poor water insolubility of our furanylnicotinamidine derivatives, the antimicrobial activities of these compounds were assessed using DMSO as a solvent. To ensure that the results obtained were reflective of the antimicrobial activity of the test compounds without interference of the solvent used, a blank experiment was performed against all tested organisms where compound-free DMSO was used to evaluate the microbial growth under the same experimental conditions. Blank experiment results showed that the tested microorganisms were able to grow in the presence of DMSO alone. Also, a literature survey showed that while DMSO can act as a chaotropic or hydrophobic stressor disordering the macromolecular systems of the cell, this holds true only at much higher concentrations than those used in our experiments employing DMSO as a solvent. ${ }^{36}$ Moreover, the concentration ranges in which the novel compounds inhibit bacterial growth are consistent with those at which inhibitors with a specific toxic mode of action (rather than stressors) inhibit the microbial growth. ${ }^{37}$

\section{Minimum inhibitory concentrations}

The MIC value is the minimum concentration of the nicotinamidine derivative at which no microbial growth is recognized. The comparison of the MICs $(\mu \mathrm{M})$ of nicotinamidine derivatives and ampicillin as a typical medication against E. coli, $P$. aeruginosa, $S$. aureus, and B. megaterium microorganisms is shown in Table 3 . Interestingly, the nicotinamidine derivatives $\mathbf{4 a}$ and $\mathbf{4 b}$ displayed an MIC value $(10 \mu \mathrm{M})$ similar to that recorded for the reference standard ampicillin against $S$. aureus. Compounds $\mathbf{4 a}$ and $\mathbf{4 b}$ showed excellent MIC values $(10 \mu \mathrm{M})$ against both $E$. coli and $S$. aureus. Equally, compound 4a exhibited the same MIC (10 $\mu \mathrm{M})$ value against $P$. aeruginosa. Additionally, the nicotinamidine derivative $\mathbf{4 g}$ displayed an excellent MIC value of $10 \mu \mathrm{M}$ against $B$. megaterium and is more potent than that recorded for the other tested nicotinamidines for the same bacterial strain. The nicotinamidine derivatives $\mathbf{4 c}-\mathbf{i}$ demonstrated good antimicrobial activities against $E$. coli with an MIC value of $15 \mu \mathrm{M}$. In addition, a good MIC value of $20 \mu \mathrm{M}$ was verified with nicotinamidines $\mathbf{4 e}, \mathbf{4 f}, \mathbf{4 h}$, and $\mathbf{4 i}$ against $P$. aeruginosa. On the other hand, the nicotinonitrile derivative 3d showed the lowest MICs in the range of 35-40 $\mu \mathrm{M}$ against the tested microorganisms.

\section{In vitro antiproliferative screening}

A series of eight compounds representing seven nicotinamidines and one nicotinonitrile were subjected to an in vitro antiproliferative screening against a panel of 60 cell lines representing nine types of human cancer. The standard practice is testing the chosen compounds at an initial high dose $(10 \mu \mathrm{M})$. Thereafter, the compounds showing a satisfactorily high MPGI, (six compounds in this research work), 
are subjected to a five-dose screen against the full panel of 60 cancer cell lines and their $\mathrm{GI}_{50}$ values are determined.

Table 4 shows the MPGI results for the initial single dose screen against the panel of 60 cancer cell lines exhibited by the seven nicotinamidines and one nicotinonitrile. First, it was found that the nicotinonitrile precursor $\mathbf{3 d}$ displayed an MPGI of 25.48 being far less active than its nicotinamidine counterpart 4d that elicited an MPGI value of -56.15 . For this reason the nicotinonitrile derivative was not progressed to the five-dose screen; these primary results clearly indicate that the presence of the amidine group plays a fundamental role in the antiproliferative activity of this class of compounds.

The structure-activity relationship findings among the seven nicotinamidine derivatives gave an insight on the structural features associated with the enhanced antiproliferative activity of this class of compounds. First, it was shown that the electron donating groups 4-methoxy, 3,5-dimethoxy-, and $p-\mathrm{N}\left(\mathrm{CH}_{3}\right)_{2}$ substitution on the phenyl ring led to a profound enhancement of antiproliferative activity as shown for the isosteres $\mathbf{4 b}, \mathbf{4 c}$, and $\mathbf{4 d}$, even enhancing the biological activity of these derivatives by being better cytostatics as in the case of $\mathbf{4 b}(\mathrm{MGPI}=33.75)$ to being cytotoxic $\mathbf{4 c}$ and 4d (MGPIs =-30.47, -56.15 , respectively) as compared to the prototype compound $4 \mathbf{a}$ (MPGI =20.02). Second, introducing withdrawing groups $p$-chloro-, $p$-fluoro-, and 3,5-dichloro- substitution on the phenyl ring led to a great enhancement of cytotoxic activity of these analogs as well. Indeed, this was the case for the $p$-chlorophenyl derivative 4e (MPGI =-74.08) and the $p$-fluorophenyl analog $4 \mathbf{f}$ (MPGI =-52.59), showing cytotoxic abilities compared to cytostatic activity for $p$-methoxyphenyl derivative $\mathbf{4 b}$. Finally, bioisosteric replacement of the 3,5-dimethoxyphenyl ring in $\mathbf{4} \mathbf{c}$ by 3,5-dichlorophenyl ring in $\mathbf{4 g}$ led to a significant increase in the MPGI value ( -49.88 for $\mathbf{4 g})$.

Table 5 displays the individual $\mathrm{GI}_{50}$ values of the six furanylnicotinamidines that were promoted for a five-dose screen due to their high antiproliferative profile demonstrated in the primary single dose screening assay. Among the tested six nicotinamidines, the most active compound was $\mathbf{4 e}$, as anticipated from the single dose screen results, showing an $\mathrm{IC}_{50}$ value less than $0.5 \mu \mathrm{M}$ against some cancer cell lines belonging to five types of cancer, namely, leukemia, non-small cell lung, colon, melanoma, and breast cancers. The most responsive to the antiproliferative effect of the tested nicotinamidine $4 \mathbf{e}$ were cancer cell lines HL-60(TB) leukemia, Colo 205 colon, and HCC-2998 colon with an $\mathrm{IC}_{50} \mathrm{~s}$ less than $0.25 \mu \mathrm{M}$. In addition, nicotinamidine $\mathbf{4 d}$ showed good activity against SR leukemia and
HT29 colon cancer cell lines with $\mathrm{GI}_{50}$ values of 0.37 and $0.47 \mu \mathrm{M}$, respectively.

Finally, Table 6 depicts the median $\mathrm{GI}_{50}$, TGI, and $\mathrm{LC}_{50}$ of the tested compounds in the five-dose screen. The most active compound, and in compliance with the primary single dose cell growth percent inhibition assay, was found to be $4 \mathbf{e}$ displaying a submicromolar $\mathrm{GI}_{50}$ value of $0.83 \mu \mathrm{M}$. All the other tested compounds exhibited $\mathrm{GI}_{50}$ values below $2 \mu \mathrm{M}$ except for $\mathbf{4 b}\left(\mathrm{GI}_{50}=5.24 \mu \mathrm{M}\right)$, which was the least active in the primary single dose screen assay. The most potent derivative was the chloro nicotinamidine $\mathbf{4 e}$ showing a micromolar TGI of $2.51 \mu \mathrm{M}$ and an $\mathrm{LC}_{50}$ of $100 \mu \mathrm{M}$. The $p$-fluoro derivative $4 \mathbf{f}$ comes second with a TGI of $2.95 \mu \mathrm{M}$ and an $\mathrm{LC}_{50}$ of $6.02 \mu \mathrm{M}$. In spite of the fact that the $p$-chloro derivative $4 \mathbf{e}$ showed high potency with regard to its growth inhibitory abilities reflected in its $\mathrm{GI}_{50}$ and TGI values $(0.83$ and $2.51 \mu \mathrm{M}$, respectively), it was the least cytotoxic in this series with an $\mathrm{LC}_{50}$ of $100 \mu \mathrm{M}$. Finally, derivative $\mathbf{4 b}$ which had the lowest MPGI ability among the six nicotinamidines selected for the five-dose screening assay was still the least potent with regard to the two measured parameters, $\mathrm{GI}_{50}$ and TGI.

\section{DNA binding and degradation}

The ability of the novel nicotinamidines $\mathbf{4 a - i}$ and nicotinonitrile derivative $\mathbf{3 d}$ to bind and degrade DNA was considered side by side to that of controls (DNA alone and DNA in DMSO) applying a horizontal electrophoresis procedure. In the present study, the tested nicotinamidines exhibited a DNA degradation effect in a dose-dependent manner, which proves their binding ability to the DNA as shown in Figure 2. Once the DNA was permitted to interrelate with the nicotinamidine derivatives at doses of $0.5,1,2,3$, and $4 \mu \mathrm{M}$, DNA cleavage was found to increase in a concentration proportional fashion as presented in Figure 2. The data show that the nicotinamidine derivative $\mathbf{4 a}$ (parent compound) is able to carry out an effective degradation for the DNA at $1 \mu \mathrm{M}$. Moreover, nicotinamidine derivatives $\mathbf{4 b}$ and $\mathbf{4 g}$ elicited effective DNA degradation at $2 \mu \mathrm{M}$. Whereas, the tested compounds $4 \mathbf{c}$, $\mathbf{4 d}$, and $4 \mathbf{i}$ at $3 \mu \mathrm{M}$ are found to exhibit strong nuclease-like activity on the genomic DNA starting at a $3 \mu \mathrm{M}$ concentration level. In addition, at $4 \mu \mathrm{M}$ concentration of the nicotinamidines $\mathbf{4 a - i}$, an efficient cleavage of DNA was observed. On the other hand, the nicotinonitrile derivative $\mathbf{3 d}$ did not reveal any degradation effect on the studied DNA at the tested concentrations range $(0.5-4 \mu \mathrm{M})$.

These results specify that DNA degradation efficiency depends on the concentration and the structural features of 

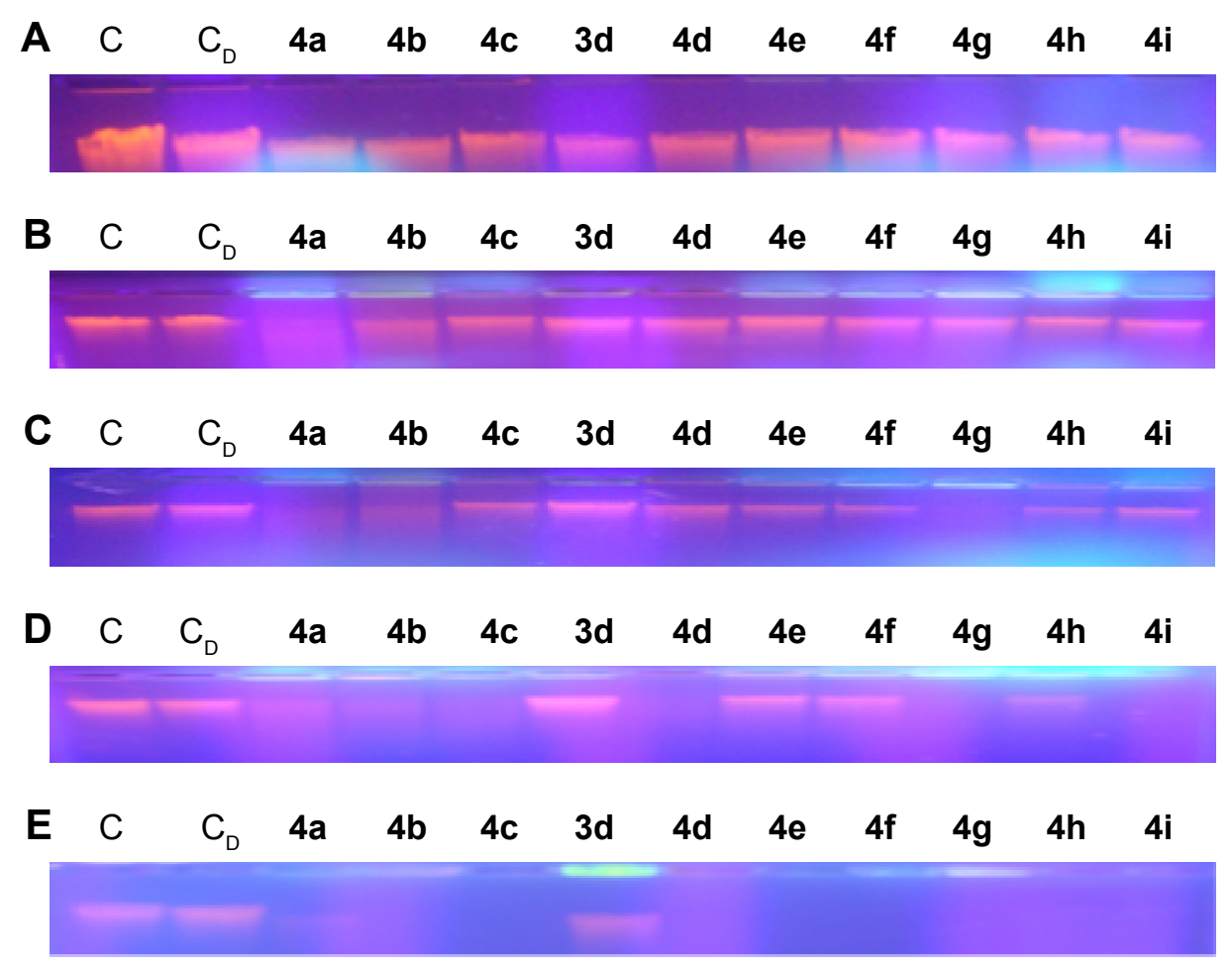

Figure 2 A figure showing the degradation effect of $0.5 \mu \mathrm{M}(\mathbf{A})$, I $\mu \mathrm{M}$ (B), $2 \mu \mathrm{M}$ (C), $3 \mu \mathrm{M}$ (D), and $4 \mu \mathrm{M}$ (E) of the novel furanylnicotinamidine derivatives (lanes 3-I2) on the genomic DNA isolated from Escherichia coli. Lanel E. coli DNA and lane 2 E. coli DNA + DMSO.

Abbreviation: DMSO, dimethylsulfoxide.

the studied compounds. It was observed that the presence of the amidine group as in derivatives $\mathbf{4} \mathbf{a}-\mathbf{i}$, compared to the cyano group of the nicotinonitrile $\mathbf{3 d}$, results in a significant DNA cleavage affinity. Thus, the newly synthesized nicotinamidines have attracted special attention as endonuclease simulators due to their significant structures. Finally, the current study validates that the nicotinamidines $\mathbf{4 a - i}$ have a substantial nuclease action toward the degradations of DNA molecule in the absence of any additional ingredients at $4 \mu \mathrm{M}$ concentration. The DNA binding and degradation action is a potential feature for the furanylnicotinamidines as promising chemotherapeutic mediators in tumor cures.

\section{Antioxidant activity}

Superoxide dismutase mimetic catalytic activity

The DNA binding studies so far revealed that the tested nicotinamidines showed good DNA binding affinity; consequently, it was considered valuable to study the antioxidant activity of this promising class of nicotinamidine derivatives. In the SOD-like activity assessment, the verified nicotinamidine derivatives participate with NBT for oxidation of the produced superoxide ions. The more effective nicotinamidines, the minor the concentration that resembles to $50 \%$ inhibition of NBT reduction.
The results presented in Table 7 report the SOD-like inhibition percent of each nicotinamidine derivative producing an effective quenching of the $\mathrm{O}_{2}^{--}$in the reaction mixture. The parent nicotinamidine derivative $4 \mathbf{a}$ and its isosteres substituted on the phenyl ring with $p$-methoxy- (4b), 3,5-dimethoxy- (4c), and $p$-dimethylamino- (4d) groups displayed a substantial SOD-like efficiency and caused an inhibition percentage of 59.7, 57.4, 54.6, and 52.9 for these compounds in the same order. Also, the halogenated derivatives $\mathbf{4 g}$ (3,5-dichloro-), $4 \mathrm{~h}$ (3,5-difluoro-), $4 \mathrm{e}$ ( $p$-chloro-),

Table 7 Superoxide dismutase mimetic catalytic activity of furanylnicotinamidine derivatives as an antioxidant enzyme

\begin{tabular}{ll}
\hline & \% inhibition \\
\hline Control & - \\
Horse radish & 69.2 \\
4a & 59.7 \\
4b & 57.4 \\
$\mathbf{4 c}$ & 54.6 \\
$\mathbf{3 d}$ & 33.6 \\
$\mathbf{4 d}$ & 52.9 \\
$\mathbf{4 e}$ & 52.0 \\
$\mathbf{4 f}$ & 51.4 \\
$\mathbf{4 g}$ & 56.1 \\
$\mathbf{4 h}$ & 52.4 \\
$\mathbf{4 i}$ & 51.5 \\
\hline
\end{tabular}


4i (2,4-difluoro-), and $\mathbf{4 f}$ ( $p$-fluoro-) exhibited a substantial SOD-like effect and contribute an inhibition percentage of $56.1 \%, 52.4 \%, 52 \%, 51.5 \%$, and $51.4 \%$, respectively. However, the nicotinonitrile compound $\mathbf{3 d}$ displayed weak SOD-like activity as denoted by an inhibition percent of $33.6 \%$.

\section{DPPH free radical scavenging activity}

The DPPH is frequently utilized as a substrate to estimate antioxidant activities of compounds. ${ }^{38}$ The $\mathrm{DPPH}^{\bullet}$ radical scavenging activity assessment is a representative assay in antioxidant activity studies and compromises a quick method for screening the radical scavenging activity of nicotinamidine compounds. The interaction of the tested nicotinamidine derivatives with steady DPPH free radical specifies their free radical scavenging capability. The tested nicotinamidines displayed antiradical activity by inhibiting DPPH radical (Figure 3). Most of the verified compounds in nicotinamidine series revealed high to moderate interaction with the DPPH radical at $2 \mu \mathrm{M}$ concentration. Significant antioxidant activities were observed for nicotinamidines and were in the following order $\mathbf{4 a}>\mathbf{4 b}>\mathbf{4 g}>\mathbf{4 c}>\mathbf{4 d}$, which all displayed more than $55 \%$ inhibition comparable to that of standard vitamin $\mathrm{C}$ tested in a parallel concentration as shown in Figure 3. It was found that substitution on the phenyl ring with $p$-methoxy-, 3,5-dimethoxy-, 3,5-dichloro-, and $p$-dimethylamino- might play a functional role in the tested nicotinamidine activity. Introduction of two chlorine atoms in $m$-positions of the phenyl ring $(\mathbf{4 g})$ increases the antioxidant activity when compared to the electron donating dimethoxy groups in the same position (4c). However, the presence of a cyano group (3d) instead of an amidine group (4d) in the same position decreased the antioxidant activity. The other nicotinamidines $\mathbf{4 e}, \mathbf{4 f}, \mathbf{4 h}$, and $\mathbf{4 i}$ exhibited good radical scavenging activity, but still less than the parent compound $\mathbf{4 a}$ as presented in Figure 3. Antioxidant activity of these compounds is related to their electron or hydrogen radical donating ability to DPPH radical. It seems that nicotinamidines retain hydrogen donating aptitudes and perform as antioxidants.

\section{NO scavenging assay}

The "novel" nicotinamidine derivatives were studied for their antioxidant property through NO scavenging activity as illustrated in Figure 4. The tested nicotinamidine derivatives demonstrated NO radical scavenging activity in the subsequent order $\mathbf{4 a}>\mathbf{4 b}>\mathbf{4 g}$. The other nicotinamidines 4c, 4d, 4e, 4f, 4h, and 4i showed good antioxidant activity, but less than the parent compound $4 \mathbf{a}$. The nicotinamidine derivative $\mathbf{4 b}$ ( $p$-MeO) exhibited greater antioxidant activity when compared to the corresponding monohalogenated compounds $4 \mathbf{e}(p-\mathrm{Cl})$ and $\mathbf{4 f}(p-\mathrm{F})$. However, the nicotinonitrile 3d exhibited feeble NO scavenging activity when compared to the control. The tested nicotinamidines possibly have the material to neutralize the effect of NO establishment and in effect possess significant ability to prevent the severe effects of dangerous $\mathrm{NO}$ generation in the living organism. NO produced from sodium nitroprusside binds with $\mathrm{O}_{2}$ to form $\mathrm{NO}_{2}$. Consequently, nicotinamidines may prevent $\mathrm{NO}_{2}$ establishment by competing with $\mathrm{O}_{2}$ to react through NO . In biological systems, NO radical has a short lifetime yet causes harm to most biomolecules, including DNA, protein,

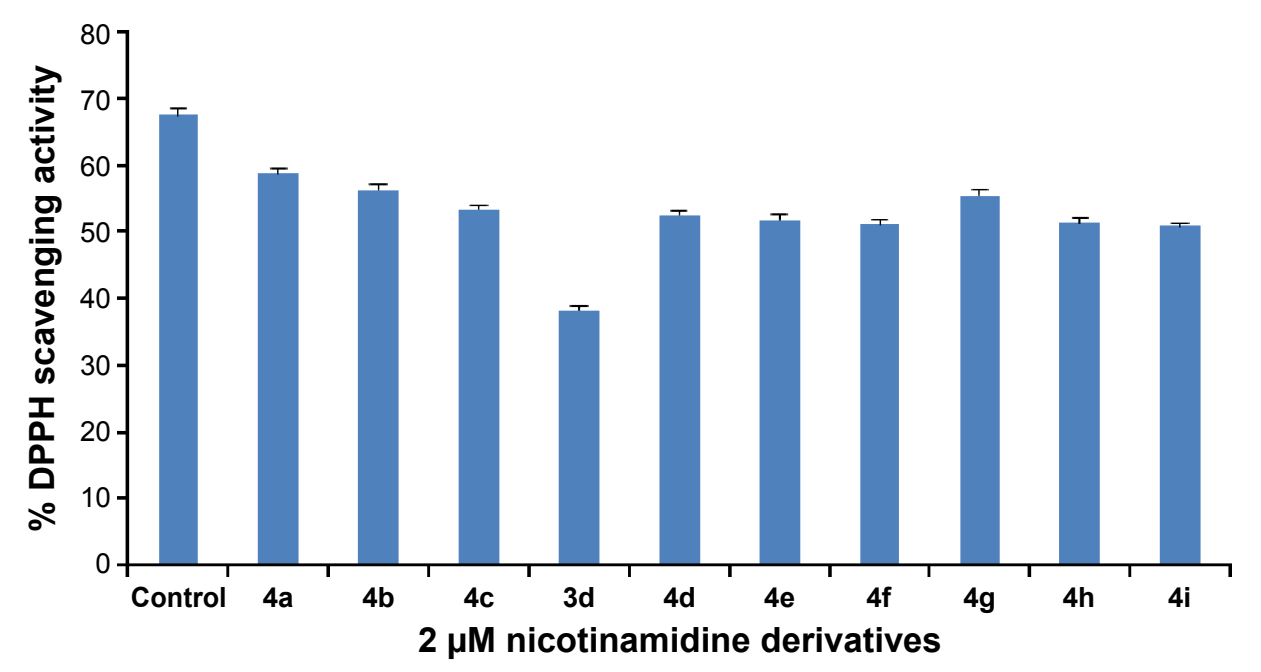

Figure 3 Antioxidant activities of the furanylnicotinamidine derivatives using 2,2-diphenyl-I-picrylhydrazyl (DPPH). 


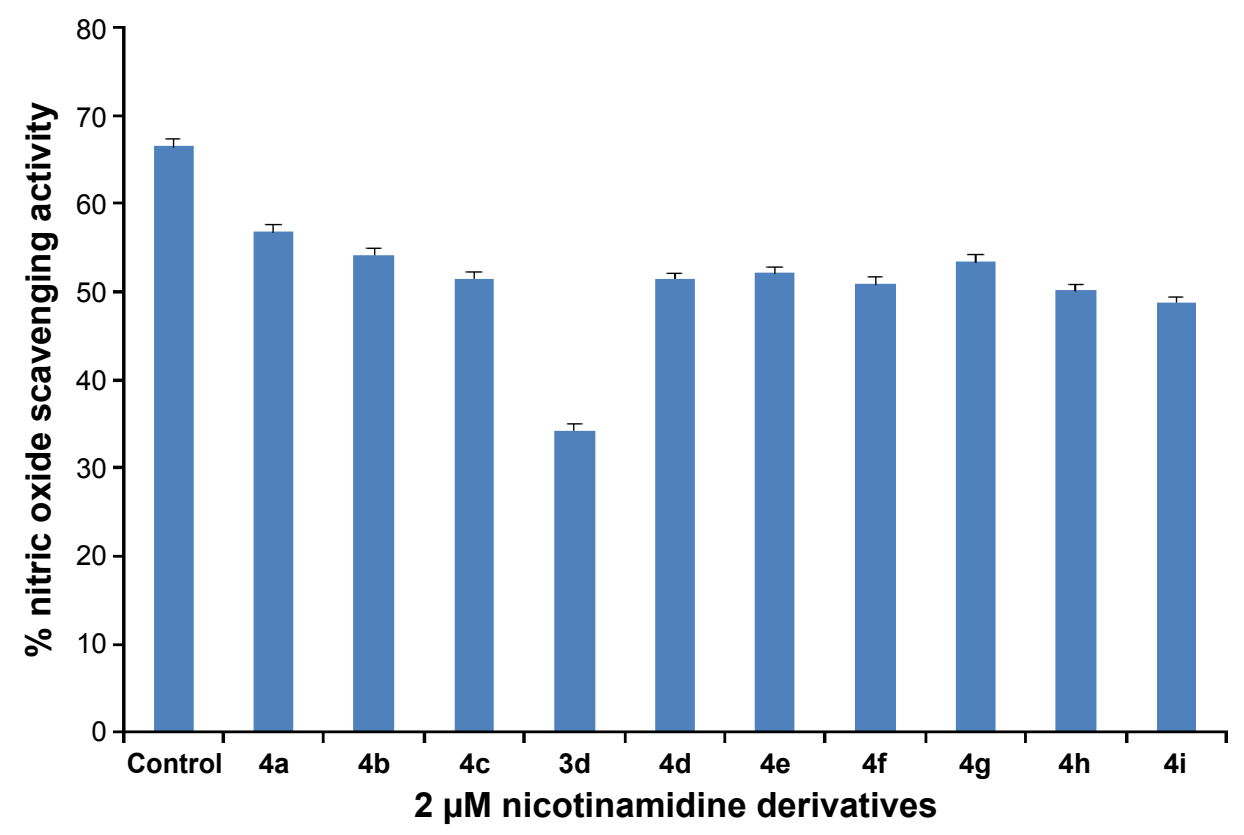

Figure 4 Antioxidant activities of the furanylnicotinamidine derivatives using nitric oxide.

and lipids, thus playing a serious role in some human diseases, including tumor and inflammation. ${ }^{39}$

\section{Conclusion}

This research endeavor has led to the synthesis of a series of substituted phenylfuranylnicotinamidines using facile synthesis procedure. Investigation into the biological activities of this class led to discovery of their anticancer, antimicrobial, DNA cleavage, and antioxidant ability. The antiproliferative activity screening was performed at NCI against 60 cancer cell lines representing nine types of cancer and revealed that the activity is linked with the substitution pattern on the terminal phenyl ring where the introduction of electron withdrawing groups was much more favorable than the electron donating ones, imparting to these derivatives not only a cytostatic property but a cytotoxic power as well. On the other hand, these nicotinamidines demonstrated good antimicrobial activity when tested against representative Gram-positive and Gram-negative bacteria. The nuclease-like activity of these nicotinamidines was also investigated and showed a dose-dependent DNA degradation ability. Finally, the antioxidant effect of the newly synthesized compounds was tested by SOD, DPPH, and NO scavenging assays and all nicotinamidines demonstrated a good antioxidant capacity. In conclusion, this class of nicotinamidines represents an easily synthesized chemotype effective in cancer management not only by virtue of their nuclease-like activity on DNA, which is useful in case of cancer cells, but also by virtue of their cytoprotective antioxidant activity in the case of normal cells.

\section{Acknowledgments}

The authors acknowledge the financial support from Deanship of Scientific Research, King Faisal University, Saudi Arabia, Project No 150122. The in vitro anticancer screening was done at the National Cancer Institute (NCI, Bethesda, MD, USA).

\section{Disclosure}

The authors report no conflicts of interest in this work.

\section{References}

1. Kennedy B, O’Connor B, Korn B, Gibbons N, O’Connor T, Keane J. Multi-drug resistant tuberculosis: experiences of two tertiary referral centres. Ir Med J. 2011;104(6):182-185.

2. Kutty N. Treating children without antibiotics in primary healthcare. Oman Med J. 2011;26(5):303-305.

3. Levy SB. Factors impacting on the problem of antibiotic resistance. J Antimicrob Chemother. 2002;49(1):25-30.

4. Lind MJ. Principles of cytotoxic chemotherapy. Medicine. 2008;36(1): 19-23.

5. Summers LA. The Bipyridinium Herbicides. London: Academic Press; 1980.

6. World Health Organization. WHO Model List of Essential Medicines. Geneva: World Health Organization; October 2013. Retrieved April 22, 2014. Available from: http://apps.who.int/iris/bitstream/10665/112738/ 1/9789240692671_eng.pdf. Accessed February 18, 2016.

7. Libman MD, Miller MA, Richards GK. Antistaphylococcal activity of pentamidine. Antimicrob Agents Chemother. 1990;34(9):1795-1796.

8. Werbovetz K. Diamidines as antitrypanosomal, antileishmanial and antimalarial agents. Curr Opin Investig Drugs. 2006;7(2):147-157.

9. Pathak MK, Dhawan D, Lindner DJ, Borden EC, Farver C, Yi T. Pentamidine is an inhibitor of PRL phosphatases with anticancer activity. Mol Cancer Ther. 2002;1(14):1255-1264. 
10. Smith J, Stewart BJ, Glaysher S, et al. The effect of pentamidine on melanoma. Anticancer Drugs. 2010;21(2):181-185.

11. Lansiaux A, Tanious F, Mishal Z, et al. Distribution of furamidine analogues in tumor cells targeting of the nucleus or mitochondria depending on the amidine substitution. Cancer Res. 2002;62(24):7219-7229.

12. Antony S, Marchand C, Stephen AG, et al. Novel high-throughput electrochemiluminescent assay for identification of human tyrosylDNA phosphodiesterase (Tdp1) inhibitors and characterization of furamidine (NSC 305831) as an inhibitor of Tdp1. Nucleic Acids Res. 2007;35(13):4474-4484.

13. Ismail MA, Brun R, Easterbrook JD, Tanious FA, Wilson WD, Boykin DW. Synthesis and antiprotozoal activity of aza-analogues of furamidine. $J$ Med Chem. 2003;46(22):4761-4769.

14. Wenzler T, Boykin DW, Ismail MA, Hall JE, Tidwell RR, Brun R. New treatment option for second-stage African sleeping sickness: in vitro and in vivo efficacy of aza analogs of DB289. Antimicrob Agents Chemother. 2009;53(10):4185-4192.

15. Thuita JK, Wolf KK, Murilla GA, et al. Safety, pharmacokinetic, and efficacy studies of oral DB868 in a first stage vervet monkey model of human African trypanosomiasis. PLoS Negl Trop Dis. 2013; 7(6):e2230.

16. Wenzler T, Yang S, Braissant O, Boykin DW, Brun R, Wang MZ. Pharmacokinetics, Trypanosoma brucei gambiense, efficacy, and time of drug action of DB829, a preclinical candidate for treatment of secondstage human African trypanosomiasis. Antimicrob Agents Chemother. 2013;57(11):5330-5343.

17. Thuita JK, Wolf KK, Murilla GA, et al. Chemotherapy of second stage human African trypanosomiasis: comparison between the parenteral diamidine DB829 and its oral prodrug DB868 in vervet monkeys. PLoS Negl Trop Dis. 2015;9(2):e0003409.

18. Youssef MM, Al-Omair MA, Ismail MA. Synthesis, DNA affinity, and antimicrobial activity of 4-substituted phenyl-2,2'-bichalcophenes and aza-analogues. Med Chem Res. 2012;21:4074-4082.

19. Hussin WA, Ismail MA, El-Sayed WM. Novel 4-substituted phenyl2,2'-bichalcophenes and aza-analogues as antibacterial agents: a structural activity relationship. Drug Des Devel Ther. 2013;7:185-193.

20. El-Sayed WM, Hussin WA, Ismail MA. Efficacy of two novel 2,2'bifurans to inhibit methicillin-resistant Staphylococcus aureus infection in male mice in comparison to vancomycin. Drug Des Devel Ther. 2012; 6:279-287.

21. Hussin WA, Ismail MA, Alzahrani AM, El-Sayed WM. Evaluation of the biological activity of novel monocationic fluoroaryl-2,2'- bichalcophenes and their analogues. Drug Des Devel Ther. 2014;8:963-972.

22. El-Sayed WM, Hussin WA. Antimutagenic and antioxidant activity of novel 4-substituted phenyl-2,2'-bichalcophenes and aza-analogs. Drug Des Devel Ther. 2013;7:73-81.

23. Ismail MA, Arafa RK, Youssef MM, El-Sayed WM. Anticancer, antioxidant activities and DNA affinity of novel monocationic bithiophenes and analogues. Drug Des Devel Ther. 2014;8:1659-1672.
24. Bauer W, Kirby WM, Sherris JC, Turck MJ. Antibiotic susceptibility testing by a standardized single disk method. Clinic Pathol. 1966; 45(4):493-496

25. Youssef MM, Mahmoud AA, Al-Faiyz YS. Antimicrobial and antioxidant activities of Artemisia abyssinica extracts and DNA degradation effects. Asian J Biochem. 2015;10(1):31-41.

26. CLSI/NCCLS. Methods for Dilution Antimicrobial Susceptibility Tests for Bacteria That Grow Aerobically, Approved Standard, 7th ed. Wayne, PA: CLSI; 2006.

27. Skehan P, Storeng R, Scudiero D, et al. New colorimetric cytotoxicity assay for anticancer-drug screening. J Natl Cancer Inst. 1990; 82(13):1107-1112.

28. McCaffrey TA, Agarwal LA, Weksler BB. A rapid fluorometric DNA assay for the measurement of cell density and proliferation in vitro. In Vitro Cell Dev Biol. 1988;24(3):247-252.

29. Monks A, Scudiero D, Skehan P, et al. Feasibility of a high-flux anticancer drug screen using a diverse panel of cultured human tumor cell lines. J Natl Cancer Inst. 1991;83(11):757-766.

30. Sayed AR, Youssef MM, Al-Faiyz YS. Synthesis, characterization, and biological evaluation of novel thiadiazoline sulfonamides and metal complexes. J Appl Sci. 2015;15(6):884-893.

31. Sambrook J, Fritsch EF, Maniatis T. Molecular Cloning: A Laboratory Manual. Cold Spring Harbor, NY: Cold Spring Harbor Laboratory Press; 1989.

32. Bridges SM, Salin ML. Distribution of iron-containing superoxide dismutase in vascular plants. Plant Physiol. 1981;68(2):275-278.

33. Al-Omair MA, Sayed AR, Youssef MM. Synthesis of novel triazoles, tetrazine, thiadiazoles and their biological activities. Molecules. 2015;20:2591-2610.

34. Green LC, Wagner DA, Glogowski J, Skipper PL, Wishnok JS, Tannenbaum SR. Analysis of nitrate, nitrite, and [15N] nitrate in biological fluids. Anal Biochem. 1982;126(1):131-138.

35. Sreejayan N, Rao MNA. Nitric oxide scavenging by curcuminoids. J Pharm Pharmacol. 1997;49(1):105-107.

36. Cray JA, Bell ANW, Bhaganna P, Mswaka AY, Timson DJ, Hallsworth JE. Hydrophobic substances induce water stress in microbial cells. Microb Biotechnol. 2013;6:453-492.

37. Cray JA, Stevenson A, Ball P, et al. Chaotropicity: a key factor in product tolerance of biofuel-producing microorganisms. Curr Opin Biotechnol. 2015;33:228-259.

38. Oyaizu M. Studies on product of browning reaction prepared from glucose amine. Jpn J Nutr. 1986;44:307-315.

39. Moncada S, Palmer RM, Higgs EA. Nitric oxide: physiology, pathophysiology, and pharmacology. Pharmacol Rev. 1991;43(2):109-142.
Drug Design, Development and Therapy

\section{Publish your work in this journal}

Drug Design, Development and Therapy is an international, peerreviewed open-access journal that spans the spectrum of drug design and development through to clinical applications. Clinical outcomes, patient safety, and programs for the development and effective, safe, and sustained use of medicines are a feature of the journal, which

\section{Dovepress}

has also been accepted for indexing on PubMed Central. The manuscript management system is completely online and includes a very quick and fair peer-review system, which is all easy to use. Visit http://www.dovepress.com/testimonials.php to read real quotes from published authors. 